\title{
PERMANENCE PROPERTIES FOR CROSSED PRODUCTS AND FIXED POINT ALGEBRAS OF FINITE GROUPS
}

\author{
CORNEL PASNICU AND N. CHRISTOPHER PHILLIPS
}

\begin{abstract}
Let $\alpha: G \rightarrow \operatorname{Aut}(A)$ be an action of a finite group $G$ on a $\mathrm{C}^{*}$ algebra $A$. We present some conditions under which properties of $A$ pass to the crossed product $C^{*}(G, A, \alpha)$ or the fixed point algebra $A^{\alpha}$. We mostly consider the ideal property, the projection property, topological dimension zero, and pure infiniteness. In many of our results, additional conditions are necessary on the group, the algebra, or the action. Sometimes the action must be strongly pointwise outer, and in a few results it must have the Rokhlin property. When $G$ is finite abelian, we prove that crossed products and fixed point algebras by $G$ preserve topological dimension zero with no condition on the action.

We give an example to show that the ideal property and the projection property do not pass to fixed point algebras (even when the group is $\mathbb{Z}_{2}$ ). The construction also gives an example of a $\mathrm{C}^{*}$-algebra $B$ which does not have the ideal property but such that $M_{2}(B)$ does have the ideal property; in fact, $M_{2}(B)$ has the projection property.
\end{abstract}

In this paper, we are interested in permanence properties for crossed products and fixed point algebras by finite groups. For the most part, we consider the following loosely related properties:

- The ideal property.

- The projection property.

- Topological dimension zero.

- Pure infiniteness for nonsimple $\mathrm{C}^{*}$-algebras.

The ideal property for a $\mathrm{C}^{*}$-algebra $A$, first defined in the introduction of 32 , requires that every ideal in $A$ be generated as an ideal by its projections. The projection property is a strengthening, introduced in Definition 1 of [20; it requires that every ideal in $A$ have an increasing approximate identity consisting of projections. (See Definition 4.8 of [5] for a variation, suitable for use with nonseparable $\mathrm{C}^{*}$-algebras.) Topological dimension zero, defined in 4] (see Definition 3.2 below), means that the topology of $\operatorname{Prim}(A)$ has a base consisting of compact open sets. The main theorem of [3] states, in effect, that if $X$ is the primitive ideal space of some separable $\mathrm{C}^{*}$-algebra, then $X$ is the primitive ideal space of an AF algebra if and only if the topology of $X$ has a base consisting of compact open sets. Pure infiniteness for not necessarily simple $\mathrm{C}^{*}$-algebras is as in Definition 4.1 of [13].

Received by the editors August 27, 2012.

2010 Mathematics Subject Classification. Primary 46L55; Secondary 46L35, 46L40.

Some of this material is based upon work of the second author, supported by the US National Science Foundation under Grants DMS-0302401, DMS-0701076, and DMS-1101742. 
According to Corollary 4.3 of 22 , if $A$ is separable and purely infinite, then the following are equivalent:

- $\mathcal{O}_{2} \otimes A$ has real rank zero.

- $\mathcal{O}_{2} \otimes A$ has the ideal property.

- $A$ has the ideal property.

- A has topological dimension zero.

The best plausible permanence results are as follows: crossed products by arbitrary discrete groups preserve pure infiniteness, while crossed products by exact actions of discrete groups preserve the other three properties when, except for a finite normal subgroup, the action is strongly pointwise outer. We do not give theorems in anything like this generality. For example, it remains unknown whether crossed products by arbitrary actions of finite groups preserve the projection property, the ideal property, or pure infiniteness.

Results on pure infiniteness of crossed products also appear in 30. They mostly have a somewhat different flavor than the results given here. Most of them assume essential freeness of the action on the equivalence classes of irreducible representations of the $\mathrm{C}^{*}$-algebra $A$, together with some kind of paradoxical decomposition for the action of $G$ on $A$, but do not assume that $A$ is purely infinite.

We summarize our results. Section 1 is devoted to Rokhlin actions of finite groups on unital $\mathrm{C}^{*}$-algebras. We show that crossed products and fixed point algebras of such actions preserve pure infiniteness, the class of countable direct limits of finite direct sums of unital Kirchberg algebras satisfying the Universal Coefficient Theorem, and the class of WB algebras.

In Section 2, we consider strongly pointwise outer actions of finite groups. We show that crossed products by such actions preserve the ideal property and the projection property. One would hope that no condition on the action would be needed, and that the result would also hold for fixed point algebras. Both together can't be true: we exhibit a pointwise outer (but not strongly pointwise outer) action of $\mathbb{Z}_{2}$ on a $\mathrm{C}^{*}$-algebra with the projection property such that the fixed point algebra does not even have the ideal property. A closely related construction gives a $\mathrm{C}^{*}$ algebra $A$ such that $M_{2}(A)$ has the projection property but $A$ does not even have the ideal property, thus giving a negative answer to a question in $[5$.

Section 3 is devoted to $\mathrm{C}^{*}$-algebras with topological dimension zero. We prove that the crossed product and fixed point algebra of an arbitrary action of a finite abelian group on such a $\mathrm{C}^{*}$-algebra again has topological dimension zero. We obtain the same result for strongly pointwise outer actions of arbitrary finite groups.

In Section 4, we consider purely infinite $\mathrm{C}^{*}$-algebras with finite primitive ideal spaces. We show that crossed products and fixed point algebras of arbitrary actions of finite groups preserve this class. They also preserve the class of purely infinite $\mathrm{C}^{*}$ algebras with composition series in which all subquotients have finite primitive ideal spaces. Thus, any example of a crossed product of a purely infinite $\mathrm{C}^{*}$-algebra by an action of a finite group which is not purely infinite must be somewhat complicated.

We use the following conventions and notation throughout this paper. Ideals in $\mathrm{C}^{*}$-algebras are always closed two sided ideals. If $\alpha: G \rightarrow \operatorname{Aut}(A)$ is an action of a group $G$ on a $C^{*}$-algebra $A$, then we denote the fixed point algebra by $A^{\alpha}$. We will usually use the same symbol $\alpha$ for the action $\left.g \mapsto \alpha_{g}\right|_{I}$ induced by $\alpha$ on an invariant ideal $I \subset A$, and similarly with invariant subalgebras and subquotients, as well as $M_{n}(A)$ and similar constructions. 
When $G$ is discrete, the standard unitary in $C^{*}(G, A, \alpha)$ (or in $M\left(C^{*}(G, A, \alpha)\right.$ ) when $A$ is not unital) corresponding to $g \in G$ will be denoted by $u_{g}$. We use the same notation for the standard unitaries in reduced crossed products. The standard conditional expectation from $C_{\mathrm{r}}^{*}(G, A, \alpha)$ to $A$ will usually be denoted by $E$.

If $A$ is a $\mathrm{C}^{*}$-algebra, then $A_{+}$denotes the set of positive elements of $A$.

We will make repeated use of the following standard fact.

Proposition 0.1. Let $A$ be a $C^{*}$-algebra, let $G$ be a compact group, and let $\alpha: G \rightarrow$ Aut $(A)$ be an action of $G$ on $A$. Then $A^{\alpha}$ is isomorphic to a corner of $C^{*}(G, A, \alpha)$.

Proof. See the Proposition in [29].

\section{Rokhlin ACTIONS OF Finite GROUPS}

The Rokhlin property for actions of finite groups is given in Definition 3.1 of [1]; see also an equivalent form in Definition 1.1 of [25, and see the beginning of Section 2 of 25] for some indication of the fairly long history of this property.

In this section we give three classes of unital $\mathrm{C}^{*}$-algebras which are preserved under formation of crossed products and fixed point algebras by actions of finite groups which have the Rokhlin property. They are the unital but not necessarily simple purely infinite $C^{*}$-algebras (as in Definition 4.1 of [13]), the unital countable direct limits of finite direct sums of Kirchberg algebras satisfying the Universal Coefficient Theorem, and the unital WB algebras (Definition 4.3 of [5]; see Definition 1.5 below) which in addition have the ideal property.

A number of other results of the same general type can be found in Sections 3 and 4 of $[17$.

The following terminology will be convenient. It is adapted from part of Definition 1.5 of [17] (without the requirement of separability) and from Definitions 2.1 and 2.2 of [5]. If $X$ is a metric space with metric $\rho$, then for $x \in X$ and $B \subset X$ we set $\operatorname{dist}(x, B)=\inf _{y \in B} \rho(x, y)$.

Definition 1.1. Let $\mathcal{C}$ be a class of $\mathrm{C}^{*}$-algebras. A strong local $\mathcal{C}$-algebra is a $\mathrm{C}^{*}$-algebra $A$ such that for every finite set $S \subset A$ and every $\varepsilon>0$, there is a $\mathrm{C}^{*}$-algebra $B \in \mathcal{C}$ and a homomorphism $\varphi: B \rightarrow A$ (not necessarily injective) such that $\operatorname{dist}(a, \varphi(B))<\varepsilon$ for all $a \in S$. We also say that $A$ can be locally approximated by $\mathcal{C}$.

We will use the following restatement of Theorem 3.2 of [17] in terms of Definition 1.1

Theorem 1.2 (Theorem 3.2 of [17]). Let $A$ be a unital $C^{*}$-algebra, let $G$ be a finite group, and let $\alpha: G \rightarrow \operatorname{Aut}(A)$ be an action with the Rokhlin property. Then $C^{*}(G, A, \alpha)$ can be locally approximated by the class of matrix algebras over corners of $A$.

Proposition 1.3. Let $A$ be a purely infinite unital $C^{*}$-algebra, let $G$ be a finite group, and let $\alpha: G \rightarrow \operatorname{Aut}(A)$ be an action with the Rokhlin property. Then $C^{*}(G, A, \alpha)$ and $A^{\alpha}$ are also purely infinite unital $C^{*}$-algebras.

We do not know of any example of any action of a finite group $G$ on a purely infinite $\mathrm{C}^{*}$-algebra $A$ such that the crossed product is not purely infinite. If $A$ is simple, then $C^{*}(G, A, \alpha)$ is always purely infinite, regardless of $\alpha$. See Corollary 4.4 of [12. In Section 4] we will give other conditions under which the crossed product of a purely infinite $\mathrm{C}^{*}$-algebra by a finite group is purely infinite. 
Proof of Proposition 1.3. By Proposition 4.17 of [13, hereditary subalgebras of purely infinite $C^{*}$-algebras are again purely infinite. It follows from Theorem 4.23 of [13] that if $B$ is purely infinite and $n \in \mathbb{Z}_{>0}$, then $M_{n}(B)$ is purely infinite. Therefore Theorem 1.2 implies that $C^{*}(G, A, \alpha)$ can be locally approximated by purely infinite $C^{*}$-algebras in the sense of Definition 1.1. The proof of Proposition 4.18 of 13 (which is stated for direct limits) shows that any $\mathrm{C}^{*}$-algebra which can be locally approximated by purely infinite $\mathrm{C}^{*}$-algebras is itself purely infinite. So $C^{*}(G, A, \alpha)$ is purely infinite.

The statement about $A^{\alpha}$ now follows from Proposition 4.17 of $[13$ and Proposition 0.1

Theorem 1.4. Let $\mathcal{C}$ be the class of unital (separable nuclear) $C^{*}$-algebras that are direct limits of sequences of finite direct sums of Kirchberg $C^{*}$-algebras satisfying the Universal Coefficient Theorem. Let $A \in \mathcal{C}$, let $G$ be a finite group, and let $\alpha: G \rightarrow \operatorname{Aut}(A)$ be an action with the Rokhlin property. Then $C^{*}(G, A, \alpha)$ and $A^{\alpha}$ are both in $\mathcal{C}$.

Proof. Let $\mathcal{C}_{0}$ be the class of $\mathrm{C}^{*}$-algebras that are finite direct sums of (not necessarily unital) Kirchberg algebras satisfying the Universal Coefficient Theorem. Clearly direct sums, corners, and quotients of unital algebras in $\mathcal{C}_{0}$ are again unital and in $\mathcal{C}_{0}$. Theorem 1.2 therefore implies that $C^{*}(G, A, \alpha)$ can be locally approximated by unital algebras in $\mathcal{C}_{0}$ in the sense of Definition 1.1. with the additional restriction that all the homomorphisms appearing in Definition 1.1 are injective. Since $C^{*}(G, A, \alpha)$ is separable, it is now easy to see that, in the sense of Definition 2.1 of [6], the crossed product $C^{*}(G, A, \alpha)$ has an exhaustive sequence of unital subalgebras in $\mathcal{C}_{0}$.

If $C^{*}(G, A, \alpha)$ were stable, we could apply Corollary 5.11 of [6] to conclude that $C^{*}(G, A, \alpha)$ is a direct limit of algebras in $\mathcal{C}_{0}$. However, according to Remark 5.13 of [6], the stability assumption in Corollary 5.11 of [6] is not necessary. We therefore conclude that $C^{*}(G, A, \alpha)$ is a direct limit of algebras in $\mathcal{C}_{0}$. Since $C^{*}(G, A, \alpha)$ is unital, it follows that $C^{*}(G, A, \alpha)$ is a direct limit of unital algebras in $\mathcal{C}_{0}$. Thus $C^{*}(G, A, \alpha) \in \mathcal{C}$.

Since corners of unital algebras in $\mathcal{C}_{0}$ are again unital and in $\mathcal{C}_{0}$, it is easy to deduce that every corner of $C^{*}(G, A, \alpha)$ is in $\mathcal{C}$. Proposition 0.1 therefore implies that $A^{\alpha} \in \mathcal{C}$.

Theorem 1.4 implies a classification result for crossed products and fixed point algebras of Rokhlin actions of finite groups on algebras in $\mathcal{C}$. Specifically, the invariant $\operatorname{Inv}_{\mathrm{u}}$ described before Lemma 4.3 of [6] classifies such algebras, because, by Theorem 5.8 of [6] and the corresponding part of Remark 5.13 of [6], this invariant classifies algebras in $\mathcal{C}$.

We now turn to WB algebras with the ideal property. We recall the definition:

Definition 1.5 (Definition 4.3 of [5]). A $C^{*}$-algebra $A$ is a $W B$ algebra if for any ideal $I \subset A$ that is generated by its projections, the extension

$$
0 \longrightarrow I \longrightarrow A \longrightarrow A / I \longrightarrow 0
$$

is quasidiagonal. That is, there is an approximate identity for $I$ consisting of projections $\left(p_{\lambda}\right)_{\lambda \in \Lambda}$ (not necessarily countable or increasing) such that $\lim \| p_{\lambda} a-$ $a p_{\lambda} \|=0$ for all $a \in A$. 
The WLB algebras of [5] will be needed.

Definition 1.6 (Definition 4.3 of [5]). A $\mathrm{C}^{*}$-algebra $A$ is said to be a $W L B$ algebra if $A$ has an approximate identity of projections (not necessarily increasing) and for every finite subset $F \subset A$ and every $\varepsilon>0$, there is a WB algebra $B$ and a homomorphism $\varphi: B \rightarrow A$ such that $\operatorname{dist}(x, \varphi(B))<\varepsilon$ for every $x \in F$ and "the projections in $F$ can be $\varepsilon$-lifted to projections in $B$ ". That is, for every projection $p \in F$, there is a projection $q \in B$ such that $\|p-\varphi(q)\|<\varepsilon$.

Lemma 1.7. Let $A$ be a unital $C^{*}$-algebra. Then $A$ is a WB algebra if and only if $A$ is a WLB algebra.

Proof. It is obvious that unital WB algebras are WLB algebras, and the reverse direction (for unital algebras) follows from Corollary 4.5 of [5].

Any LB algebra (Definition 2.2 of [21]) is a WLB algebra, so that unital LB algebras are WB algebras. The class of LB algebras contains the GAH algebras (Definition 2.1 of [19]), and thus the AH algebras.

Lemma 1.8. Let $A$ be a $W B$ algebra, and let $p \in A$ be a projection. Then $p A p$ is a WB algebra.

Proof. Let $J \subset p A p$ be an ideal which is generated by its projections. We have to show that the extension

$$
0 \longrightarrow J \longrightarrow p A p \longrightarrow p A p / J \longrightarrow 0
$$

is quasidiagonal. Set $I=\overline{A J A} \subset A$, an ideal in $A$ which is generated by its projections, and which satisfies $p I p=J$. The corresponding extension is quasidiagonal by hypothesis, so Lemma 3.3 of [5] gives the desired conclusion.

Proposition 1.9. Let $A$ be a unital $W B$ algebra with the ideal property, let $G$ be a finite group, and let $\alpha: G \rightarrow \operatorname{Aut}(A)$ be an action with the Rokhlin property. Then $C^{*}(G, A, \alpha)$ and $A^{\alpha}$ are also unital $W B$ algebras with the ideal property.

Since the algebras involved are unital, Lemma 1.7 implies that we get an equivalent statement by replacing all instances of "WB algebra" with "WLB algebra".

The method of proof does not work for the ideal property alone, since, by Example 2.8 below, the ideal property is not preserved by passing to corners. It follows from Proposition 4.16 of [26] and Corollary 2.6 below that crossed products by Rokhlin actions of finite groups on unital $\mathrm{C}^{*}$-algebras do preserve the ideal property. It is not clear what happens with fixed point algebras. Example 2.7 shows that the hypotheses of Corollary 2.6 are too weak to ensure that the fixed point algebra has the ideal property.

Proof of Proposition 1.9. We first prove that $C^{*}(G, A, \alpha)$ is a WLB algebra with the ideal property. We show that $C^{*}(G, A, \alpha)$ is a WLB algebra by proving the local approximation property in Definition 1.6. The approximating algebras $B$ will also have the ideal property, so it will follow from Corollary 2.5 of [5] that $C^{*}(G, A, \alpha)$ has the ideal property.

Thus, let $F \subset C^{*}(G, A, \alpha)$ be finite and let $\varepsilon>0$. Choose $\rho>0$ with $\rho \leq \varepsilon$ and so small that whenever $C$ is a $\mathrm{C}^{*}$-algebra, $D \subset C$ is a subalgebra, $p \in C$ is a projection, and $\operatorname{dist}(p, D)<\rho$, then there is a projection $q \in D$ such that $\|p-q\|<\varepsilon$. Use Theorem 1.2 to find $n \in \mathbb{Z}_{>0}$, a projection $f \in M_{n}(A)$, and a 
homomorphism $\varphi: f M_{n}(A) f \rightarrow C^{*}(G, A, \alpha)$ (not necessarily injective) such that $\operatorname{dist}\left(a, \varphi\left(f M_{n}(A) f\right)\right)<\rho$ for all $a \in F$. Set $B=f M_{n}(A) f / \operatorname{ker}(\varphi)$, and let $\bar{\varphi}: B \rightarrow$ $C^{*}(G, A, \alpha)$ be the map induced by $\varphi$.

It follows from the proof of Lemma 4.14 of [5] that $M_{n}(A)$ is a WB algebra, and obviously $M_{n}(A)$ has the ideal property. Therefore $f M_{n}(A) f$ is a WB algebra by Lemma 1.8, and has the ideal property by Proposition 4.9 of [5]. Since $f M_{n}(A) f$ has the ideal property, Remark 4.2 of [5] implies that $B$ is a WB algebra, and clearly $B$ has the ideal property. The choice of $\rho$ implies that for every $x \in F$ we have $\operatorname{dist}(x, \bar{\varphi}(B))<\varepsilon$. Since $\bar{\varphi}$ is injective, it also follows that for every projection $p \in F$, there is a projection $q \in B$ such that $\|p-\varphi(q)\|<\varepsilon$. This completes the proof that $C^{*}(G, A, \alpha)$ is a WLB algebra with the ideal property.

Lemma 1.7 now implies that $C^{*}(G, A, \alpha)$ is a WB algebra.

Proposition 0.1 exhibits $A^{\alpha}$ as a corner of $C^{*}(G, A, \alpha)$, so it follows from Proposition 4.9 of [5] that $A^{\alpha}$ is also a unital WB algebra with the ideal property.

\section{Strongly POINTWISE OUTER ACTIONS AND THE IDEAL AND PROJECTION PROPERTIES}

Strongly pointwise outer actions (see Definition 2.1 below) are those for which no group element $g \neq 1$ is inner on any $g$-invariant subquotient. When the $\mathrm{C}^{*}$-algebra is simple, this condition is just pointwise outerness.

In this section we show that if $\alpha$ is a strongly pointwise outer action of a finite group $G$ on a $\mathrm{C}^{*}$-algebra $A$, then $A$ separates the ideals in $C^{*}(G, A, \alpha)$. It follows that such actions are hereditarily saturated and their crossed products preserve the ideal and projection properties.

It seems plausible that crossed products and fixed point algebras of arbitrary actions of finite groups should preserve the ideal and projection properties. We have not settled the question for crossed products, but we show by example that the statement about fixed point algebras is false, even when $G=\mathbb{Z}_{2}$. Our construction further produces a $\mathrm{C}^{*}$-algebra $B$ such that $M_{2}(B)$ has the projection property but $B$ does not even have the ideal property. This gives a negative answer to Question 6.8 of [5].

Definition 2.1 (Definition 4.11 of $[26$ ). An action $\alpha: G \rightarrow \operatorname{Aut}(A)$ is said to be strongly pointwise outer if, for every $g \in G \backslash\{1\}$ and any two $\alpha_{g}$-invariant ideals $I \subset J \subset A$ with $I \neq J$, the automorphism of $J / I$ induced by $\alpha_{g}$ is outer.

Definition $2.2([31])$. Let $\alpha: G \rightarrow \operatorname{Aut}(A)$ be an action of a discrete group $G$ on a $C^{*}$-algebra $A$. We say that $A$ separates the ideals in the reduced crossed product $C_{\mathrm{r}}^{*}(G, A, \alpha)$ (or in $C^{*}(G, A, \alpha)$ when $G$ is amenable) if each ideal of $C_{\mathrm{r}}^{*}(G, A, \alpha)$ has the form $C_{\mathrm{r}}^{*}(G, I, \alpha)$ for some $\alpha$-invariant ideal $I \subset A$.

By Proposition 7.7.9 of [23], for every $\alpha$-invariant ideal $I \subset A$, the obvious map $C_{\mathrm{r}}^{*}(G, I, \alpha) \rightarrow C_{\mathrm{r}}^{*}(G, A, \alpha)$ is injective. Its image is clearly an ideal.

Theorem 2.3. Let $G$ be a finite group, let $A$ be a $C^{*}$-algebra, and let $\alpha: G \rightarrow$ $\operatorname{Aut}(A)$ be a strongly pointwise outer action. Then $A$ separates the ideals in $C^{*}(G, A, \alpha)$.

Proof. Let $J$ be an ideal in $C^{*}(G, A, \alpha)$. Set $I=J \cap A$. We claim that $J$ is an $\alpha$-invariant ideal in $A$ such that $C^{*}(G, I, \alpha) \subset J$. For $\alpha$-invariance, let $a \in I$ and 
$g \in G$. Then $\alpha_{g}(a)=u_{g} a u_{g}^{*}$ is in both $J$ and $A$. If $a \in I$ and $g \in G$, then $a \in J$, so $a u_{g} \in J$. Thus $C^{*}(G, I, \alpha) \subset J$. The claim is proved.

Suppose $C^{*}(G, I, \alpha) \neq J$. Let $B=A / I$, and let $\pi: A \rightarrow B$ be the quotient map. Deviating from our usual convention (to avoid confusion below), let $\beta: G \rightarrow \operatorname{Aut}(B)$ be the induced action on the quotient. Let $\rho: C^{*}(G, A, \alpha) \rightarrow C^{*}(G, B, \beta)$ be the map induced by $\pi$. Then $\operatorname{ker}(\rho)=C^{*}(G, I, \alpha)$. Let $L=\rho(J)$. Then $L$ is a nonzero ideal of $C^{*}(G, B, \beta)$, but $L \cap B=\{0\}$. It follows from Theorem 1.1 of [28] that there is $g \in G \backslash\{1\}$ such that $\beta_{g}$ is partly inner in the sense of [28]; that is, there is some nonzero $\beta_{g}$-invariant ideal $T \subset B$ such that the restriction of $\beta_{g}$ to $T$ is inner (in $M(T)$ ). This contradicts the assumption that $\alpha$ is strongly pointwise outer.

Examples 4.13 and 4.14 of [26] show that several weaker versions of Definition 2.1 do not suffice for the conclusion of Theorem 2.3. In particular, one must consider subquotients (not just ideals and quotients), and one must consider subquotients invariant under subgroups, not just subquotients invariant under the whole group.

Corollary 2.4. Let $G$ be a finite group, let $A$ be a $C^{*}$-algebra, and let $\alpha: G \rightarrow$ $\operatorname{Aut}(A)$ be a strongly pointwise outer action. Then $\alpha$ is hereditarily saturated (Definition 7.2.2 of [24]) and the strong Connes spectrum $\widetilde{\Gamma}(\alpha)$ (Definition 1.2(b) of [8]) is equal to $\widehat{G}$, the space of unitary equivalence classes of irreducible representations of $G$.

Proof. This follows from Theorem 2.3 and [8, as described in Theorem 5.11 of [26].

As an another immediate consequence of Theorem 2.3, we obtain the following result, which we originally proved directly from the definition.

Corollary 2.5. Let $G$ be a finite group, let $A$ be a unital $C^{*}$-algebra, and let $\alpha: G \rightarrow \operatorname{Aut}(A)$ be an action with the Rokhlin property. Then $A$ separates the ideals in $C^{*}(G, A, \alpha)$.

Proof. Use Proposition 4.16 of [26] and Theorem 2.3.

Corollary 2.6. Crossed products by strongly pointwise outer actions of finite groups preserve the ideal property and the projection property.

Proof. Let $\alpha: G \rightarrow \operatorname{Aut}(A)$ be a strongly pointwise outer action of a finite group $G$ on a $\mathrm{C}^{*}$-algebra $A$.

Assume that $A$ has the ideal property. Let $J \subset C^{*}(G, A, \alpha)$ be an ideal. By Theorem 2.3. there is a $G$-invariant ideal $I \subset A$ such that $J=C^{*}(G, I, \alpha)$. By hypothesis, $I$ is generated by its projections, and it is then easy to see that $C^{*}(G, I, \alpha)$ is generated by its projections.

The argument for the projection property is the same.

We do not know of any example of any action of a finite group on a $\mathrm{C}^{*}$-algebra with the ideal property such that the crossed product does not have the ideal property. Similarly, we do not know of any example of any action of a finite group on a $\mathrm{C}^{*}$-algebra with the projection property such that the crossed product does not have the projection property.

The following example shows that the ideal and projection properties do not pass to fixed point algebras of actions of finite groups. In fact, we produce an example of a pointwise outer (but not strongly pointwise outer) action of $\mathbb{Z}_{2}$ on a $\mathrm{C}^{*}$-algebra 
with the projection property such that the fixed point algebra does not even have the ideal property.

Example 2.7. Let $D$ be the unital Kirchberg algebra satisfying the Universal Coefficient Theorem and with $K_{0}(D)=0$ and $K_{1}(D) \cong \mathbb{Z}_{2}$. For any $\mathrm{C}^{*}$-algebra $E$, let $Q(E)=M(E) / E$, the corona algebra. It follows from Theorem 3.3 of 34 that $Q(K \otimes D)$ is simple, and from Theorem 1.3 of [33] that $Q(K \otimes D)$ is purely infinite. Since $K_{*}(M(K \otimes D))=0$ (by Proposition 12.2.1 of [2]), the six term exact sequence in K-theory gives $K_{0}(Q(K \otimes D)) \cong \mathbb{Z}_{2}$ and $K_{1}(Q(K \otimes D))=0$. Let $\pi: M(K \otimes D) \rightarrow Q(K \otimes D)$ be the quotient map. Since $1 \in Q(K \otimes D)$ is equal to $\pi(1)$, and $K_{0}(M(K \otimes D))=0$, it follows that [1] $=0$ in $K_{0}(Q(K \otimes D))$.

Since $Q(K \otimes D)$ is purely infinite and simple, there is a projection $e \in Q(K \otimes D)$ whose class in $K_{0}(Q(K \otimes D))$ is the nontrivial element, and any two such projections are Murray-von Neumann equivalent. Since $[1-e]=[1]-[e]=-[e]=[e]$, there is $s \in Q(K \otimes D)$ such that $s^{*} s=e$ and $s s^{*}=1-e$. Set $v=s+s^{*}$, which is a unitary in $Q(K \otimes D)$ such that $v^{*}=v$ and $v e v^{*}=1-e$. Since $v$ is a selfadjoint unitary, it is in the identity component of the unitary group of $Q(K \otimes D)$, and therefore there is a unitary $u \in M(K \otimes D)$ such that $\pi(u)=v$. (We can't choose $u$ to satisfy $u^{2}=1$.)

Set $C=\mathbb{C} e+\mathbb{C}(1-e)$, which is a subalgebra of $Q(K \otimes D)$ with $C \cong \mathbb{C} \oplus \mathbb{C}$. Define a subalgebra $B \subset M(K \otimes D) \oplus M(K \otimes D)$ by

$$
B=\left\{\left(b_{1}, b_{2}\right) \in M(K \otimes D) \oplus M(K \otimes D): \pi\left(b_{1}\right), \pi\left(b_{2}\right) \in C \text { and } \pi\left(b_{1}\right)=\pi\left(b_{2}\right)\right\} .
$$

Let $\kappa: B \rightarrow C$ be the map $\kappa\left(b_{1}, b_{2}\right)=\pi\left(b_{1}\right)$. Then there is a short exact sequence

$$
0 \longrightarrow K \otimes D \oplus K \otimes D \longrightarrow B \stackrel{\kappa}{\longrightarrow} C \longrightarrow 0 .
$$

Define $\gamma \in \operatorname{Aut}(C)$ by

$$
\gamma\left(\lambda_{1} e+\lambda_{2}(1-e)\right)=\lambda_{2} e+\lambda_{1}(1-e)
$$

for $\lambda_{1}, \lambda_{2} \in \mathbb{C}$. Then $v c v^{*}=\gamma(c)$ for all $c \in C$. Define $\beta: B \rightarrow B$ by

$$
\beta\left(b_{1}, b_{2}\right)=\left(u b_{2} u^{*}, u^{*} b_{1} u\right) .
$$

We claim that if $\left(b_{1}, b_{2}\right) \in B$, then $\beta\left(b_{1}, b_{2}\right)$ really is in $B$. Indeed, using $v=v^{*}$ and $\pi\left(b_{1}\right)=\pi\left(b_{2}\right)$ at the second step, we have

$$
\pi\left(u b_{2} u^{*}\right)=v \pi\left(b_{2}\right) v^{*}=v^{*} \pi\left(b_{1}\right) v=\pi\left(u b_{1} u^{*}\right) .
$$

Also, $\pi\left(u b_{2} u^{*}\right) \in C$ because $v C v^{*}=C$. This proves the claim. One checks immediately that $\beta^{2}=\mathrm{id}_{B}$, so $\beta$ is invertible Also, since for $c \in C$ we have $v c v^{*}=\gamma(c)$, for $\left(b_{1}, b_{2}\right) \in B$ we get

$$
(\kappa \circ \beta)\left(b_{1}, b_{2}\right)=\pi\left(u b_{2} u^{*}\right)=v \pi\left(b_{2}\right) v^{*}=v \pi\left(b_{1}\right) v^{*}=(\gamma \circ \kappa)\left(b_{1}, b_{2}\right) .
$$

Let $\varphi \in \operatorname{Aut}(K \otimes D \oplus K \otimes D)$ be the restriction of $\beta$. Then $\varphi, \beta$, and $\gamma$ all define actions of $\mathbb{Z}_{2}$, which we denote by the same letters, and the exact sequence (2.1) is equivariant.

We take crossed products in (2.1). We have $C^{*}\left(\mathbb{Z}_{2}, C, \gamma\right) \cong M_{2}$. The automorphism $\sigma$ of $K \otimes D \oplus K \otimes D$ defined by

$$
\sigma\left(b_{1}, b_{2}\right)=\left(u^{*} b_{1} u, b_{2}\right)
$$

satisfies

$$
\left(\sigma \circ \beta \circ \sigma^{-1}\right)\left(b_{1}, b_{2}\right)=\left(b_{2}, b_{1}\right)
$$


so, defining $\rho=\sigma \circ \beta \circ \sigma^{-1}$, we have

$$
C^{*}\left(\mathbb{Z}_{2}, K \otimes D \oplus K \otimes D, \rho\right) \cong M_{2} \otimes K \otimes D .
$$

Letting $A=C^{*}\left(\mathbb{Z}_{2}, B, \beta\right)$, we therefore get a short exact sequence, equivariant for the dual actions:

$$
0 \longrightarrow M_{2} \otimes K \otimes D \longrightarrow A \longrightarrow M_{2} \longrightarrow 0 .
$$

We claim that $A$ has the projection property. From the sequence (2.2), we see that $A$ has only two nonzero ideals, namely $M_{2} \otimes K \otimes D$ and $A$ itself. The algebra $A$ is unital, and $M_{2} \otimes K \otimes D$ has an increasing approximate identity consisting of projections because $D$ is unital. The claim follows.

We claim that $B$ does not have the ideal property. To prove this, we set $J=$ $\kappa^{-1}(\mathbb{C} e)$, which is an ideal in $B$, and show that $J$ is not generated by its projections. It suffices to show that if $p \in J$ is a projection, then $\kappa(p)=0$. Write $p=\left(p_{1}, p_{2}\right)$ with $p_{1}, p_{2} \in M(K \otimes D)$. Then $\pi\left(p_{1}\right)$ is a projection in $\mathbb{C} e$, and so $\pi\left(p_{1}\right)=e$ or $\pi\left(p_{1}\right)=0$. However, $\pi\left(p_{1}\right)=e$ is ruled out by $\left[p_{1}\right]=0$ in $K_{0}(M(K \otimes D))$ and $[e] \neq 0$ in $K_{0}(Q(K \otimes D))$. So $\kappa\left(p_{1}, p_{2}\right)=\pi\left(p_{1}\right)=0$. This proves the claim.

Let $\alpha=\widehat{\beta}$ be the dual action, and identify $\widehat{\mathbb{Z}_{2}}$ with $\mathbb{Z}_{2}$. Then $A^{\alpha}=B$. Accordingly, we have an action of $\mathbb{Z}_{2}$ on a $\mathrm{C}^{*}$-algebra $A$ with the projection property (and, in particular, the ideal property) such that $A^{\alpha}$ does not have the ideal property (and, in particular, does not have the projection property).

As preparation for proving that $\alpha$ is pointwise outer, we claim that the center of $A$ is $\mathbb{C} \cdot 1$. It is clear that $M_{2} \otimes K \otimes D$ is an essential ideal in $A$, so we can identify $A$ with a subalgebra of $M\left(M_{2} \otimes K \otimes D\right)$ which contains $M_{2} \otimes K \otimes D$. Since $M_{2} \otimes K \otimes D$ is simple,

$$
\left\{z \in M\left(M_{2} \otimes K \otimes D\right): z b=b z \text { for all } b \in M_{2} \otimes K \otimes D\right\}=\mathbb{C} \cdot 1 .
$$

The claim follows.

Now let $\varphi \in \operatorname{Aut}(A)$ be the automorphism of order two which generates the action $\alpha$; we prove that $\varphi$ is outer. If not, there is a unitary $u \in A$ such that $\varphi(a)=u a u^{*}$ for all $a \in A$. Then $\varphi(u)=u$, so $u^{2} a u^{-2}=\varphi^{2}(a)=a$ for all $a \in A$. By the previous paragraph, there is $\lambda_{0} \in \mathbb{C}$ with $\left|\lambda_{0}\right|=1$ such that $u^{2}=\lambda_{0} \cdot 1$. Choose $\lambda \in \mathbb{C}$ such that $\lambda^{-2}=\lambda_{0}$ and set $v=\lambda u$. Then $v^{2}=1$ and $\varphi(a)=v a v^{*}$ for all $a \in A$. So $\alpha$ is an inner action. Therefore $A \oplus A \cong C^{*}\left(\mathbb{Z}_{2}, A, \alpha\right) \cong M_{2}(B)$. Since $B$ has no nontrivial direct sum decomposition, this contradicts innerness of $\varphi$, and shows that $\alpha$ is pointwise outer.

Question 6.8 of [5] asked the following. Let $A$ be a $\mathrm{C}^{*}$-algebra, let $n \in \mathbb{Z}_{>0}$, and suppose that $M_{n}(A)$ has the ideal property. Does it follow that $A$ has the ideal property? It is also natural to ask the same question for the projection property in place of the ideal property. The following example provides negative answers to both questions.

Example 2.8. We use the notation of Example 2.7 throughout. We claim that $M_{2}(B)$ does have the projection property. Thus, $M_{2}(B)$ can have the projection property when $B$ does not even have the ideal property.

To prove the claim, we consider the nonzero ideals in $B$. There are six of them: $K \otimes D \oplus\{0\}, \quad\{0\} \oplus K \otimes D, \quad K \otimes D \oplus K \otimes D, \quad \kappa^{-1}(\mathbb{C} e), \quad \kappa^{-1}(\mathbb{C}(1-e)), \quad B$. For each ideal $J$ on this list, we need to show that $M_{2}(J)$ has an increasing approximate identity consisting of projections. For all but $\kappa^{-1}(\mathbb{C} e)$ and $\kappa^{-1}(\mathbb{C}(1-e))$, 
this is immediate. Moreover, with $\beta$ as in Example 2.7. we have $\beta\left(\kappa^{-1}(\mathbb{C} e)\right)=$ $\kappa^{-1}(\mathbb{C}(1-e))$. Therefore it suffices to consider $\kappa^{-1}(\mathbb{C} e)$.

By abuse of notation, given a homomorphism $\varphi: E \rightarrow F$, we use the same letter for the corresponding homomorphism from $M_{2}(E)$ to $M_{2}(F)$.

Define $w \in M_{2}(Q(K \otimes D))$ by

$$
w=\left(\begin{array}{cc}
e & s \\
s^{*} & 1-e
\end{array}\right) .
$$

One checks that $w$ is a selfadjoint unitary, and that

$$
w\left(\begin{array}{ll}
e & 0 \\
0 & e
\end{array}\right) w^{*}=\left(\begin{array}{ll}
1 & 0 \\
0 & 0
\end{array}\right) .
$$

Since $w$ is a selfadjoint unitary, $w$ is in the identity component of the unitary group of $M_{2}(Q(K \otimes D))$. Therefore there is a unitary $y \in M_{2}(M(K \otimes D))$ such that $\pi(y)=w$. Define $p \in M_{2}(M(K \otimes D))$ by

$$
p=y^{*}\left(\begin{array}{ll}
1 & 0 \\
0 & 0
\end{array}\right) y \text {. }
$$

Then $p$ is a projection and

$$
\pi(p)=\left(\begin{array}{cc}
e & 0 \\
0 & e
\end{array}\right)
$$

Moreover, $1-p$ is unitarily equivalent to $1 \oplus 0$, from which it follows that

$$
(1-p) M_{2}(K \otimes D)(1-p) \cong K \otimes D
$$

has an increasing approximate identity $\left(f_{n}\right)_{n \in \mathbb{Z}_{>0}}$ consisting of projections.

Define $q_{n} \in M_{2}\left(\kappa^{-1}(\mathbb{C} e)\right)$ by

$$
q_{n}=\left(p+f_{n}, p+f_{n}\right) .
$$

We claim that $\left(q_{n}\right)_{n \in \mathbb{Z}_{>0}}$ is an approximate identity for $M_{2}\left(\kappa^{-1}(\mathbb{C} e)\right)$. We begin by observing that, for any $a \in M_{2}\left(\pi^{-1}(\mathbb{C} e)\right)$, we have $\pi((1-p) a)=0$, so

$$
(1-p) a a^{*}(1-p) \in(1-p) M_{2}(K \otimes D)(1-p) .
$$

Thus

$$
\begin{aligned}
& \|\left[f_{n}(1-p) a-\right.(1-p) a] \cdot\left[f_{n}(1-p) a-(1-p) a\right]^{*} \| \\
& \leq\left\|f_{n}(1-p) a a^{*}(1-p)-(1-p) a a^{*}(1-p)\right\| \cdot\left\|f_{n}\right\| \\
& \quad+\left\|f_{n}(1-p) a a^{*}(1-p)-(1-p) a a^{*}(1-p)\right\|,
\end{aligned}
$$

which converges to zero as $n \rightarrow \infty$. So

$$
\lim _{n \rightarrow \infty}\left\|f_{n}(1-p) a-(1-p) a\right\|=0 .
$$

Using $f_{n}(1-p)=f_{n}$, for $a \in M_{2}\left(\pi^{-1}(\mathbb{C} e)\right)$ we therefore get

$$
\lim _{n \rightarrow \infty}\left(\left(p+f_{n}\right) a-a\right)=\lim _{n \rightarrow \infty}\left(f_{n}(1-p) a-(1-p) a\right)=0 .
$$

Since

$$
M_{2}\left(\kappa^{-1}(\mathbb{C} e)\right)=\left\{\left(b_{1}, b_{2}\right) \in M_{2}\left(\pi^{-1}(\mathbb{C} e)\right): \pi\left(b_{1}\right)=\pi\left(b_{2}\right)\right\},
$$

we immediately get $\lim _{n \rightarrow \infty}\left(q_{n} b-b\right)=0$ for all $b \in M_{2}\left(\kappa^{-1}(\mathbb{C} e)\right)$. Taking adjoints, we also get $\lim _{n \rightarrow \infty}\left(b q_{n}-b\right)=0$ for all $b \in M_{2}\left(\kappa^{-1}(\mathbb{C} e)\right)$. So $\left(q_{n}\right)_{n \in \mathbb{Z}_{>0}}$ is an approximate identity, as desired. This completes the proof that $M_{2}(B)$ has the projection property. 


\section{TOPOLOGICAL DIMENSION ZERO}

In this section, we prove that arbitrary crossed products by actions of finite abelian groups preserve the property of topological dimension zero of [4]. (See Definition 3.2) See the introduction for some of the significance of this condition. Our proof depends on duality. It seems plausible that noncommutative duality could be used to extend the result to nonabelian groups, but we have not succeeded in carrying this out. We do show (with a much easier proof) that the result holds for nonabelian groups if in addition the algebra separates the ideals in the crossed product.

In the following, topological spaces need not be Hausdorff unless otherwise specified. A compact set is one which has the Heine-Borel property, regardless of whether or not it is closed or Hausdorff. (This property is sometimes called quasicompactness.) We repeat these statements for emphasis in some of the definitions and lemmas. We give the following definition to make our terminology clear.

Definition 3.1 (Compare with 3.3.8 of [7]). Let $X$ be a not necessarily Hausdorff topological space. We say that $X$ is locally compact if for every $x \in X$ and every open set $U \subset X$ such that $x \in U$, there exists a compact (but not necessarily closed) subset $Y \subset X$ such that $x \in \operatorname{int}(Y) \subset Y \subset U$.

Equivalantly, the compact neighborhoods of every point $x \in X$ form a neighborhood base at $x$.

Definition 3.2 (Remark 2.5(vi) of [4]). Let $X$ be a locally compact but not necessarily Hausdorff topological space. We say that $X$ has topological dimension zero if for every $x \in X$ and every open set $U \subset X$ such that $x \in U$, there exists a compact open (but not necessarily closed) subset $Y \subset X$ such that $x \in Y \subset U$. We further say that a $C^{*}$-algebra $A$ has topological dimension zero if $\operatorname{Prim}(A)$ has topological dimension zero.

We do not write $\operatorname{dim}(X)=0$ or use similar notation, because other values of the topological dimension are not defined for spaces of this generality.

Lemma 3.3. Let $A$ be a $C^{*}$-algebra $A$ with topological dimension zero. Then every hereditary subalgebra $B$ of $A$ also has topological dimension zero.

Proof. Theorem 5.5.5 of [16] implies that $\operatorname{Prim}(B)$ is homeomorphic to an open subset of $\operatorname{Prim}(A)$, and clearly an open subset of a space with topological dimension zero again has topological dimension zero.

Definition 3.4. Let $X$ be a not necessarily Hausdorff topological space. A compact open exhaustion of $X$ is an increasing net $\left(Y_{\lambda}\right)_{\lambda \in \Lambda}$ of compact open subsets $Y_{\lambda} \subset X$ such that $X=\bigcup_{\lambda \in \Lambda} Y_{\lambda}$.

Lemma 3.5. Let $X$ be a not necessarily Hausdorff topological space. The following are equivalent:

(1) X has a compact open exhaustion.

(2) For every $x \in X$ there exists a compact open (but not necessarily closed) subset $Y \subset X$ such that $x \in Y$.

Proof. That (11) implies (2) is obvious. For the reverse, for every $x \in X$ choose a compact open subset $Z_{x} \subset X$ such that $x \in Z_{x}$. Take $\Lambda$ to be the collection of all finite subsets of $X$, and for $\lambda \in \Lambda$ set $Y_{\lambda}=\bigcup_{x \in \lambda} Z_{x}$. 
Lemma 3.6. Let $X$ be a locally compact but not necessarily Hausdorff topological space. The following are equivalent:

(1) $X$ has topological dimension zero.

(2) Every open subset of $X$ has a compact open exhaustion.

(3) The compact open sets form a base for the topology of $X$.

Proof. The equivalence of (1) and (2) follows from Lemma 3.5. The equivalence of (11) and (3) is the definition of a base for a topology.

The proof of the following lemma is adapted from the proof of Proposition 2.6 of [4].

Lemma 3.7. Let $X$ be a locally compact but not necessarily Hausdorff topological space. Let $U \subset X$ be open. Suppose that $X \backslash U$ has a compact open exhaustion (in the relative topology) and $U$ has a compact open exhaustion. Then $X$ has a compact open exhaustion.

Proof. We verify condition (2) in Lemma 3.5. So let $x \in X$. We need to find a compact open subset $Y \subset X$ such that $x \in Y$.

If $x \in U$, then, since open subsets of $U$ are open in $X$, we simply apply the hypothesis on $U$. So assume that $x \in X \backslash U$. By Lemma 3.5, there is a compact subset $Z \subset X \backslash U$ such that $x \in Z$ and $Z$ is open in $X \backslash U$ in the relative topology. Choose an open subset $W \subset X$ such that $Z=W \cap(X \backslash U)$. Since $X$ is locally compact, for every $z \in Z$ there is a compact set $R(z) \subset X$ such that

$$
z \in \operatorname{int}(R(z)) \subset R(z) \subset W .
$$

Since $Z$ is compact, there are $n \in \mathbb{Z}_{>0}$ and $z_{1}, z_{2}, \ldots, z_{n} \in Z$ such that

$$
\operatorname{int}\left(R\left(z_{1}\right)\right), \operatorname{int}\left(R\left(z_{2}\right)\right), \ldots, \operatorname{int}\left(R\left(z_{n}\right)\right)
$$

cover $Z$. Set $R=\bigcup_{k=1}^{n} R\left(z_{k}\right)$. Then $R$ is compact and $Z \subset \operatorname{int}(R) \subset R \subset W$. Since $W \cap(X \backslash U)=Z$, it follows that $R \backslash \operatorname{int}(R) \subset U$. Also, $R \backslash \operatorname{int}(R)$ is closed in $R$ in the relative topology of $R$, and is hence compact. Since $U$ has a compact open exhaustion, there is a compact open subset $C \subset U$ such that $R \backslash \operatorname{int}(R) \subset C$. Set $Y=C \cup R$. Then $Y$ is compact because $C$ and $R$ are compact. Also, $Y$ is open because $Y=C \cup \operatorname{int}(R)$. Since $x \in R \subset Y$, the proof is complete.

Lemma 3.8. Let $X$ be a topological space with a compact open exhaustion. Then every closed subset of $X$ has a compact open exhaustion in the relative topology.

Proof. Let $Y \subset X$ be closed. Choose an increasing net $\left(L_{\lambda}\right)_{\lambda \in \Lambda}$ of compact open subsets $L_{\lambda} \subset X$ such that $X=\bigcup_{\lambda \in \Lambda} L_{\lambda}$. For $\lambda \in \Lambda$, set $M_{\lambda}=L_{\lambda} \cap Y$. Then $M_{\lambda}$ is compact because $Y$ is closed and, even when the space is not Hausdorff, the intersection of a compact set and a closed set is compact. Clearly $\bigcup_{\lambda \in \Lambda} M_{\lambda}=Y$.

Definition 3.9 (See page 53 of [22]). An ideal $I$ in a $\mathrm{C}^{*}$-algebra $A$ is said to be compact if whenever $\left(I_{\lambda}\right)_{\lambda \in \Lambda}$ is an increasing net of ideals in $A$ such that $I=$ $\overline{\bigcup_{\lambda \in \Lambda} I_{\lambda}}$, then there is $\lambda \in \Lambda$ such that $I=I_{\lambda}$.

Lemma 3.10. Let $A$ be a $C^{*}$-algebra and let $I \subset A$ be an ideal. Then $I$ is compact if and only if $\operatorname{Prim}(I)$ is a compact open subset of $\operatorname{Prim}(A)$.

Proof. Compactness of $I$ is equivalent to the statement that whenever $\left(U_{\lambda}\right)_{\lambda \in \Lambda}$ is an increasing net of open subsets of $\operatorname{Prim}(A)$ such that $\operatorname{Prim}(I)=\bigcup_{\lambda \in \Lambda} U_{\lambda}$, then there is $\lambda \in \Lambda$ such that $\operatorname{Prim}(I)=U_{\lambda}$. 
Lemma 3.11. Let $\alpha: G \rightarrow \operatorname{Aut}(A)$ be an action of a finite group $G$ on a $C^{*}$ algebra $A$. Let $J$ be an $\alpha$-invariant ideal in $A$. Then $J \subset \overline{A\left(J \cap A^{\alpha}\right)}$.

Proof. Set $n=\operatorname{card}(G)$. Choose an approximate identity $\left(a_{\lambda}\right)_{\lambda \in \Lambda}$ for $J$. For $\lambda \in \Lambda$, define

$$
b_{\lambda}=\frac{1}{n} \sum_{g \in G} \alpha_{g}\left(a_{\lambda}\right) .
$$

Since $J$ is $G$-invariant, $\left(\alpha_{g}\left(a_{\lambda}\right)\right)_{\lambda \in \Lambda}$ is also an approximate identity for $J$ for every $g \in G$. So $\left(b_{\lambda}\right)_{\lambda \in \Lambda}$ is an approximate identity for $J$. Moreover, $b_{\lambda} \in A^{\alpha}$ for all $\lambda \in \Lambda$. If $x \in J$, we therefore have $x=\lim _{\lambda \in \Lambda} x b_{\lambda} \in \overline{A\left(J \cap A^{\alpha}\right)}$. This completes the proof.

Lemma 3.12. Let $\alpha: G \rightarrow \operatorname{Aut}(A)$ be an action of a finite group $G$ on a $C^{*}$ algebra $A$. Let $J$ be an $\alpha$-invariant ideal in $A$. If $J \cap A^{\alpha}$ is a compact ideal in $A^{\alpha}$, then $J$ is a compact ideal in $A$.

Proof. Set $I=J \cap A^{\alpha}$. Let $\left(J_{\lambda}\right)_{\lambda \in \Lambda}$ be an increasing net of ideals in $A$ such that $J=\overline{\bigcup_{\lambda \in \Lambda} J_{\lambda}}$. We must find $\lambda$ such that $J_{\lambda}=J$. For $\lambda \in \Lambda$, define $I_{\lambda}=J_{\lambda} \cap A^{\alpha}$, which is an ideal in $A^{\alpha}$.

We claim that $\overline{\bigcup_{\lambda \in \Lambda} I_{\lambda}}=I$. To prove this, let $a \in I$ and let $\varepsilon>0$. For $g \in G$, we have

$$
a=\alpha_{g}(a) \in \alpha_{g}(J)=\overline{\bigcup_{\lambda \in \Lambda} \alpha_{g}\left(J_{\lambda}\right)},
$$

so there are $\lambda_{g} \in \Lambda$ and $x_{g} \in \alpha_{g}\left(J_{\lambda_{g}}\right)$ such that $0 \leq x_{g} \leq 1$ and $\left\|a x_{g}-a\right\|<n^{-1} \varepsilon$. Choose $\lambda \in \Lambda$ such that for all $g \in G$ we have $\lambda \geq \lambda_{g}$.

Choose a bijection $k:\{1,2, \ldots, n\} \rightarrow G$. Define

$$
x=\frac{1}{n} \sum_{g \in G} \alpha_{g}\left(x_{k(1)} x_{k(2)} \cdots x_{k(n)}\right) \text {. }
$$

We have

$$
x_{k(1)} x_{k(2)} \cdots x_{k(n)} \in \bigcap_{g \in G} \alpha_{g}\left(J_{\lambda}\right) .
$$

Since $\bigcap_{g \in G} \alpha_{g}\left(J_{\lambda}\right)$ is $\alpha$-invariant, it follows that $x \in \bigcap_{g \in G} \alpha_{g}\left(J_{\lambda}\right)$. In particular, $x \in J_{\lambda}$. Clearly $x \in A^{\alpha}$, so $x \in I_{\lambda}$. Also, $\left\|a x_{k(1)}-a\right\|<n^{-1} \varepsilon$, so

$$
\left\|a x_{k(1)} x_{k(2)}-a\right\| \leq\left\|a x_{k(1)}-a\right\| \cdot\left\|x_{k(2)}\right\|+\left\|a x_{k(2)}-a\right\|<\frac{\varepsilon}{n}+\frac{\varepsilon}{n}=\frac{2 \varepsilon}{n} .
$$

An induction argument shows that

$$
\left\|a x_{k(1)} x_{k(2)} \cdots x_{k(n)}-a\right\|<\varepsilon .
$$

Since $\alpha_{g}^{-1}(a)=a$ for all $g \in G$, we also have

$$
\left\|a \cdot \alpha_{g}\left(x_{k(1)} x_{k(2)} \cdots x_{k(n)}\right)-a\right\|<\varepsilon
$$

for all $g \in G$. Therefore $\|a x-a\|<\varepsilon$. Since $a x \in I_{\lambda}$, we have $\operatorname{dist}\left(a, I_{\lambda}\right)<\varepsilon$. Since $\varepsilon>0$ is arbitrary, it follows that $a \in \overline{\bigcup_{\lambda \in \Lambda} I_{\lambda}}$, proving the claim.

Since $I$ is compact, there is $\lambda \in \Lambda$ such that $I=I_{\lambda}$. Using Lemma 3.11, we then get $J \subset \overline{A I}=\overline{A I_{\lambda}} \subset J_{\lambda}$. Thus $J_{\lambda}=J$, showing that $J$ is compact. 
Lemma 3.13. Let $\alpha: G \rightarrow \operatorname{Aut}(A)$ be an action of a finite group $G$ on a $C^{*}$ algebra A. Suppose that whenever $I \subset A^{\alpha}$ is a compact ideal, then $\overline{A I A} \cap A^{\alpha}$ is a compact ideal in $A^{\alpha}$. Let $J$ be an $\alpha$-invariant ideal in $A$. If $\operatorname{Prim}\left(J \cap A^{\alpha}\right) \subset$ $\operatorname{Prim}\left(A^{\alpha}\right)$ has a compact open exhaustion, then $\operatorname{Prim}(J) \subset \operatorname{Prim}(A)$ has a compact open exhaustion.

Proof. By Lemma 3.10, it suffices to find an increasing net $\left(J_{\lambda}\right)_{\lambda \in \Lambda}$ of compact ideals in $A$ such that $J=\overline{\bigcup_{\lambda \in \Lambda} J_{\lambda}}$. Lemma 3.10 implies that there is an increasing net $\left(I_{\lambda}\right)_{\lambda \in \Lambda}$ of compact ideals in $A^{\alpha}$ such that $J \cap A^{\alpha}=\overline{\bigcup_{\lambda \in \Lambda} I_{\lambda}}$. For $\lambda \in \Lambda$, set $J_{\lambda}=\overline{A I_{\lambda} A}$. By hypothesis, $\overline{A I_{\lambda} A} \cap A^{\alpha}$ is compact. So Lemma 3.12 implies that $J_{\lambda}$ is compact. Clearly $J_{\lambda} \subset J$. Since $J$ is $\alpha$-invariant, Lemma 3.11 implies the first step of the following calculation:

$$
J \subset \overline{A\left(J \cap A^{\alpha}\right)} \subset \overline{A\left(J \cap A^{\alpha}\right) A} \subset \overline{A\left(\bigcup_{\lambda \in \Lambda} I_{\lambda}\right) A}=\overline{\bigcup_{\lambda \in \Lambda} A I_{\lambda} A}=\overline{\bigcup_{\lambda \in \Lambda} J_{\lambda}} .
$$

This completes the proof.

Theorem 3.14. Let $\alpha: G \rightarrow \operatorname{Aut}(A)$ be an action of a finite group $G$ on a $C^{*}$ algebra $A$. Suppose that $A^{\alpha}$ has topological dimension zero. Suppose also that whenever $I \subset A^{\alpha}$ is a compact ideal, then $\overline{A I A} \cap A^{\alpha}$ is a compact ideal in $A^{\alpha}$. Then $A$ has topological dimension zero.

Proof. We verify that $\operatorname{Prim}(A)$ satisfies condition (2) of Lemma 3.6. So let $V \subset$ $\operatorname{Prim}(A)$ be open. Let $I \subset A$ be the corresponding ideal. We will use Lemma 5.3.3 of [24]. Thus, for $S \subset G$ with $1 \in S$, we define $\alpha$-invariant ideals $I_{S}, I_{S}^{-} \subset A$ by

$$
I_{S}=\sum_{g \in G} \alpha_{g}\left(\bigcap_{h \in S} \alpha_{h}(I)\right) \text { and } I_{S}^{-}=\sum_{g \in G \backslash S} I_{S \cup\{g\}} .
$$

Then $I_{G} \subset I \subset I_{\{1\}}$ (Lemma 5.3.3(3) of [24]).

We prove by downwards induction on the set $S$ that $\operatorname{Prim}\left(I_{S} \cap I\right)$ has a compact open exhaustion. We start with $S=G$. Since $I_{G} \cap I=I_{G}$ is $\alpha$-invariant, this follows from Lemma 3.13

Suppose now $S$ is given, with $1 \in S$, and we know that $\operatorname{Prim}\left(I_{T} \cap I\right)$ has a compact open exhaustion for all $T \subset G$ such that $S \subset T$ and $S \neq T$. We have

$$
I_{S}^{-} \cap I=\sum_{g \in G \backslash S}\left(I_{S \cup\{g\}} \cap I\right),
$$

so

$$
\operatorname{Prim}\left(I_{S}^{-} \cap I\right)=\bigcup_{g \in G \backslash S} \operatorname{Prim}\left(I_{S \cup\{g\}} \cap I\right) .
$$

It is easily checked that the union of open subsets with compact open exhaustions again has a compact open exhaustion. So $\operatorname{Prim}\left(I_{S}^{-} \cap I\right)$ has a compact open exhaustion.

Since $I_{S}$ is $G$-invariant, Lemma 3.13 implies that $\operatorname{Prim}\left(I_{S}\right)$ has a compact open exhaustion, and now Lemma 3.8 implies that $\operatorname{Prim}\left(I_{S} / I_{S}^{-}\right)$has a compact open exhaustion. Lemma 5.3.3(5) of [24] implies that $\left[\left(I_{S} \cap I\right)+I_{S}^{-}\right] / I_{S}^{-}$is a direct summand in $I_{S} / I_{S}^{-}$. Therefore Prim $\left(\left[\left(I_{S} \cap I\right)+I_{S}^{-}\right] / I_{S}^{-}\right)$has a compact open exhaustion by Lemma 3.8. We have a short exact sequence

$$
0 \longrightarrow I_{S}^{-} \cap I \longrightarrow I_{S} \cap I \longrightarrow\left[\left(I_{S} \cap I\right)+I_{S}^{-}\right] / I_{S}^{-} \longrightarrow 0,
$$


so Lemma 3.7 implies that $\operatorname{Prim}\left(I_{S} \cap I\right)$ has a compact open exhaustion. This completes the induction step.

Since $I_{\{1\}} \cap I=I$, we conclude that $V=\operatorname{Prim}(I)$ has a compact open exhaustion.

The hypothesis in Theorem 3.14 involving $\overline{A I A} \cap A^{\alpha}$ is annoying. (It enters via Lemma 3.13, )

Question 3.15. Let $\alpha: G \rightarrow \operatorname{Aut}(A)$ be an action of a finite group $G$ on a $\mathrm{C}^{*}$ algebra $A$. Suppose that $I \subset A^{\alpha}$ is a compact ideal. Does it follow that $\overline{A I A} \cap A^{\alpha}$ is also a compact ideal in $A^{\alpha}$ ?

We note that $\overline{A I A} \cap A^{\alpha}$ need not equal $I$. Indeed, there are many cases in which $A$ is simple but $A^{\alpha}$ is not. (For example, take $A=L\left(l^{2}(G)\right.$ ) with the action given by conjugation by the regular representation of $G$.) Then for any nontrivial ideal $I \subset A^{\alpha}$, one gets $\overline{A I A} \cap A^{\alpha}=A^{\alpha} \neq I$.

If $A$ is a crossed product of $A^{\alpha}$ by an action of an abelian group, then Question 3.15 has a positive answer. Accordingly, we get a result for abelian groups. To keep the notation simple, we start with a separate lemma.

Lemma 3.16. Let $\alpha: G \rightarrow \operatorname{Aut}(A)$ be an action of a finite group $G$ on a $C^{*}$ algebra $A$. Let $I \subset A$ be an ideal. Then

$$
\overline{C^{*}(G, A, \alpha) I C^{*}(G, A, \alpha)} \cap A=\sum_{g \in G} \alpha_{g}(I) .
$$

Proof. For $g \in G$, let $u_{g} \in M\left(C^{*}(G, A, \alpha)\right)$ be the standard unitary corresponding to $g$, as in the introduction. Then

$$
\alpha_{g}(I)=u_{g} I u_{g}^{*} \subset \overline{M\left(C^{*}(G, A, \alpha)\right) I M\left(C^{*}(G, A, \alpha)\right)}=\overline{C^{*}(G, A, \alpha) I C^{*}(G, A, \alpha)} .
$$

This proves one of the desired inclusions.

For the other, set $L=\sum_{g \in G} \alpha_{g}(I)$. Then $L$ is $\alpha$-invariant, so $C^{*}(G, L, \alpha)$ is an ideal in $C^{*}(G, A, \alpha)$ which clearly contains $I$. Also $C^{*}(G, L, \alpha) \cap A=L$. Therefore

$$
\overline{C^{*}(G, A, \alpha) I C^{*}(G, A, \alpha)} \cap A \subset C^{*}(G, L, \alpha) \cap A=L=\sum_{g \in G} \alpha_{g}(I) .
$$

This completes the proof.

Theorem 3.17. Let $\alpha: G \rightarrow \operatorname{Aut}(A)$ be an action of a finite abelian group $G$ on a $C^{*}$-algebra $A$. Suppose that $A$ has topological dimension zero. Then $C^{*}(G, A, \alpha)$ and $A^{\alpha}$ have topological dimension zero.

Proof. We first consider $C^{*}(G, A, \alpha)$. Let $\widehat{\alpha}: \widehat{G} \rightarrow \operatorname{Aut}\left(C^{*}(G, A, \alpha)\right)$ be the dual action. Then $C^{*}(G, A, \alpha)^{\widehat{\alpha}} \cong A$, and so has topological dimension zero.

We check that $\widehat{\alpha}$ satisfies the second hypothesis of Theorem 3.14. Let $I \subset A$ be a compact ideal. Applying Lemma 3.16 we get

$$
\overline{C^{*}(G, A, \alpha) I C^{*}(G, A, \alpha)} \cap A=\sum_{g \in G} \alpha_{g}(I) .
$$

The right hand side is compact by Lemma 3.10 and because the union of finitely many compact sets is compact.

Theorem 3.14 now implies that $C^{*}(G, A, \alpha)$ has topological dimension zero.

The result for $A^{\alpha}$ now follows from Lemma 3.3 and Proposition 0.1 
We now turn to an action $\alpha$ of a general finite group $G$ on a $\mathrm{C}^{*}$-algebra $A$, but under the assumption that $A$ separates the ideals in $C^{*}(G, A, \alpha)$. The proof is much more straightforward, but the machinery developed above seems to be of little help.

Proposition 3.18. Let $\alpha: G \rightarrow \operatorname{Aut}(A)$ be an action of a finite group $G$ on a $C^{*}$ algebra A. Assume that $A$ separates the ideals in $C^{*}(G, A, \alpha)$. Suppose that $A$ has topological dimension zero. Then $C^{*}(G, A, \alpha)$ and $A^{\alpha}$ have topological dimension zero.

Part of the proof works for reduced crossed products by actions of discrete groups, so we give it in that generality. Let $\alpha: G \rightarrow \operatorname{Aut}(A)$ be an action of a discrete group $G$ on a $\mathrm{C}^{*}$-algebra $A$. Let $E: C_{\mathrm{r}}^{*}(G, A, \alpha) \rightarrow A$ be the canonical conditional expectation (as in the introduction). It is immediate that if $I \subset A$ is an $\alpha$-invariant ideal, then

$$
E\left(C_{\mathrm{r}}^{*}(G, I, \alpha)\right)=I .
$$

It follows that for $\alpha$-invariant ideals $I_{1}, I_{2} \subset A$, we have

$$
I_{1} \subset I_{2} \text { if and only if } C_{\mathrm{r}}^{*}\left(G, I_{1}, \alpha\right) \subset C_{\mathrm{r}}^{*}\left(G, I_{2}, \alpha\right) .
$$

Lemma 3.19. Let $\alpha: G \rightarrow \operatorname{Aut}(A)$ be an action of a discrete group $G$ on a $C^{*}$ algebra A. Suppose $A$ separates the ideals in $C_{\mathrm{r}}^{*}(G, A, \alpha)$. Let I be an $\alpha$-invariant ideal of $A$. If $I$ is compact, then $C_{\mathrm{r}}^{*}(G, I, \alpha)$ is also compact.

Proof. Let $\left(J_{\lambda}\right)_{\lambda \in \Lambda}$ be an increasing net of ideals in $C_{\mathrm{r}}^{*}(G, A, \alpha)$ such that

$$
C_{\mathrm{r}}^{*}(G, I, \alpha)=\overline{\bigcup_{\lambda \in \Lambda} J_{\lambda}}
$$

By hypothesis, there are $\alpha$-invariant ideals $I_{\lambda}$ such that $J_{\lambda}=C_{\mathrm{r}}^{*}\left(G, I_{\lambda}, \alpha\right)$ for all $\lambda \in \Lambda$. By (3.2), we have $I_{\lambda} \subset I$ for all $\lambda \in \Lambda$, and moreover $\left(I_{\lambda}\right)_{\lambda \in \Lambda}$ is increasing. By (3.1) and because $E$ is continuous, we have

$$
\begin{aligned}
I= & E\left(C_{\mathrm{r}}^{*}(G, I, \alpha)\right)=E\left(\overline{\bigcup_{\lambda \in \Lambda} C_{\mathrm{r}}^{*}\left(G, I_{\lambda}, \alpha\right)}\right) \\
& \subset \overline{E\left(\bigcup_{\lambda \in \Lambda} C_{\mathrm{r}}^{*}\left(G, I_{\lambda}, \alpha\right)\right)}=\overline{\bigcup_{\lambda \in \Lambda} E\left(C_{\mathrm{r}}^{*}\left(G, I_{\lambda}, \alpha\right)\right)}=\overline{\bigcup_{\lambda \in \Lambda} I_{\lambda}} \subset I .
\end{aligned}
$$

Thus $I=\overline{\bigcup_{\lambda \in \Lambda} I_{\lambda}}$. Since $I$ is compact, there is $\lambda \in \Lambda$ such that $I=I_{\lambda}$. Then $C_{\mathrm{r}}^{*}(G, I, \alpha)=C_{\mathrm{r}}^{*}\left(G, I_{\lambda}, \alpha\right)=J_{\lambda}$. This shows that $C_{\mathrm{r}}^{*}(G, I, \alpha)$ is compact.

Proof of Proposition 3.18. We first consider $C^{*}(G, A, \alpha)$.

It follows from Lemma 3.6 and Lemma 3.10 that a $\mathrm{C}^{*}$-algebra $D$ has topological dimension zero if and only if every ideal in $D$ is the closure of the union of an increasing net of compact ideals.

So let $J$ be an arbitrary ideal in $C^{*}(G, A, \alpha)$. By hypothesis, there is an $\alpha$ invariant ideal $I \subset A$ such that $J=C^{*}(G, I, \alpha)$. Since $A$ has topological dimension zero, there is an increasing net $\left(I_{\lambda}\right)_{\lambda \in \Lambda}$ of compact ideals of $A$ such that $I=$ $\overline{\bigcup_{\lambda \in \Lambda} I_{\lambda}}$. For $\lambda \in \Lambda$, define $L_{\lambda}=\sum_{g \in G} \alpha_{g}\left(I_{\lambda}\right)$. Then $\left(L_{\lambda}\right)_{\lambda \in \Lambda}$ is an increasing net of $\alpha$-invariant ideals and $\overline{\bigcup_{\lambda \in \Lambda} L_{\lambda}}=I$. Since a finite union of compact sets is compact, Lemma 3.10 implies that $L_{\lambda}$ is compact for all $\lambda \in \Lambda$. The ideals 
$C^{*}\left(G, L_{\lambda}, \alpha\right)$ are compact by Lemma 3.19, By (3.2), these ideals are increasing and satisfy

$$
\overline{\bigcup_{\lambda \in \Lambda} C^{*}\left(G, L_{\lambda}, \alpha\right)}=C^{*}(G, I, \alpha) .
$$

This completes the proof for $C^{*}(G, A, \alpha)$.

The result for $A^{\alpha}$ now follows from Lemma 3.3 and Proposition 0.1

Corollary 3.20. Let $\alpha: G \rightarrow \operatorname{Aut}(A)$ be a strongly pointwise outer action of a finite group $G$ on a $C^{*}$-algebra $A$. Suppose that $A$ has topological dimension zero. Then $C^{*}(G, A, \alpha)$ and $A^{\alpha}$ have topological dimension zero.

Proof. It follows from Theorem 2.3 that $A$ separates the ideals in $C^{*}(G, A, \alpha)$. Now apply Proposition 3.18 .

\section{Purely infinite $\mathrm{C}^{*}$-Algebras with finite primitive SPeCtrum}

Let $A$ be a purely infinite $\mathrm{C}^{*}$-algebra, and let $\alpha: G \rightarrow \operatorname{Aut}(A)$ be an action of a finite group $G$ on $A$. We know of no examples in which $C^{*}(G, A, \alpha)$ and $A^{\alpha}$ are not purely infinite, but we have not been able to prove that they are. The main result of this section is that if $A$ has a composition series in which all the subquotients have finite primitive ideal spaces, then $C^{*}(G, A, \alpha)$ and $A^{\alpha}$ must be purely infinite. A counterexample to the general statement, if it exists, must therefore be fairly complicated.

We record the following standard fact.

Lemma 4.1. Let $A$ be a $C^{*}$-algebra. Then $A$ has finitely many ideals if and only if $\operatorname{Prim}(A)$ is finite.

Proof. The forward implication is trivial. The reverse follows from the fact that ideals in $A$ are in one to one correspondence with open subsets of $\operatorname{Prim}(A)$.

Lemma 4.2. Let $A$ be a $C^{*}$-algebra and let $I$ be an ideal of $A$. The following are equivalent:

(1) $\operatorname{Prim}(A)$ is finite.

(2) $\operatorname{Prim}(I)$ and $\operatorname{Prim}(A / I)$ are finite.

Proof. The result is immediate from the fact that $\operatorname{Prim}(A)$ is the (not necessarily topological) disjoint union of $\operatorname{Prim}(I)$ and $\operatorname{Prim}(A / I)$.

Corollary 4.3. Let $I$ and $J$ be ideals in a $C^{*}$-algebra $A$ which have finite primitive ideal spaces. Then $\operatorname{Prim}(I+J)$ is finite.

Proof. Consider the short exact sequence of $\mathrm{C}^{*}$-algebras,

$$
0 \longrightarrow I \longrightarrow I+J \longrightarrow J /(I \cap J) \longrightarrow 0 .
$$

The space $\operatorname{Prim}(J /(I \cap J))$ is finite by one direction in Lemma 4.2, so $\operatorname{Prim}(I+J)$ is finite by the other direction.

Lemma 4.4. Let $I$ and $J$ be ideals in a $C^{*}$-algebra $A$. Assume that $I$ and $J$ are purely infinite. Then $I+J$ is purely infinite. 
Proof. Consider the short exact sequence of $\mathrm{C}^{*}$-algebras

$$
0 \longrightarrow I \longrightarrow I+J \longrightarrow J /(I \cap J) \longrightarrow 0 .
$$

Pure infiniteness passes to quotients (Proposition 4.3 of [13]), so $J /(I \cap J)$ is purely infinite. Extensions of purely infinite $\mathrm{C}^{*}$-algebras are purely infinite (Theorem 4.19 of [13]), so $I+J$ is purely infinite.

Lemma 4.5. Let $\alpha: G \rightarrow \operatorname{Aut}(A)$ be an action of a finite group $G$ on a $C^{*}$ algebra $A$. Suppose that $A$ is the sum of orthogonal ideals which are permuted transitively by the action of $G$. Let $I$ be one of these ideals, and let $H$ be the subgroup of elements of $G$ which carry $I$ into itself, so that $H$ acts on $I$. Set $n=\operatorname{card}(G / H)$. Then $C^{*}(G, A, \alpha) \cong M_{n}\left(C^{*}(H, I, \alpha)\right)$.

Proof. As described after the proof of Proposition 2.3 of [28], this is a special case of Theorem 2.13(i) of [9].

Lemma 4.6. Let $\alpha: G \rightarrow \operatorname{Aut}(A)$ be an action of a finite group $G$ on a $C^{*}$ algebra $A$. Assume that $A$ is $\alpha$-simple. Then $C^{*}(G, A, \alpha)$ is a finite direct sum of simple $C^{*}$-algebras. Moreover, if in addition $A$ is purely infinite, then $C^{*}(G, A, \alpha)$ is a finite direct sum of simple purely infinite $C^{*}$-algebras.

Proof. Since $A$ is $\alpha$-simple, the discussion at the beginning of Section 3 of 28 ] implies that the hypotheses of Lemma 4.5 hold, with, in addition, $I$ being simple. Set $n=\operatorname{card}(G / H)$. Then $C^{*}(G, A, \alpha) \cong M_{n}\left(C^{*}(H, I, \alpha)\right)$. Theorem 3.1 of 28 now implies that $C^{*}(G, A, \alpha)$ is a finite direct sum of simple $\mathrm{C}^{*}$-algebras.

If $I$ is unital, the second part conclusion now follows from Theorem 4.5 of 12 . However, the proof of this theorem (including the proofs of Theorem 4.2 and Corollary 4.4 of [12; the nonunital case of Theorem 2.1 of [12] is the first part of the present lemma) also works for nonunital $\mathrm{C}^{*}$-algebras, provided one uses multiplier algebras at the appropriate places.

Theorem 4.7. Let $\alpha: G \rightarrow \operatorname{Aut}(A)$ be an action of a finite group $G$ on a $C^{*}$-algebra $A$. Assume that $A$ has finitely many $\alpha$-invariant ideals. Then $\operatorname{Prim}\left(C^{*}(G, A, \alpha)\right)$ is finite. Moreover, if in addition $A$ is purely infinite, then $C^{*}(G, A, \alpha)$ is purely infinite.

Proof. Since $A$ has finitely many $\alpha$-invariant ideals, every $\alpha$-invariant ideal $I \subset A$ contains a maximal strictly smaller $\alpha$-invariant ideal $J$. In particular, $I / J$ is $\alpha$ simple. An induction argument therefore shows that $A$ has a finite composition series $\left(I_{k}\right)_{0 \leq k \leq n}$ consisting of $\alpha$-invariant ideals such that $I_{k+1} / I_{k}$ is $\alpha$-simple for $k=0,1, \ldots, n-1$.

Since $G$ is amenable, for $k=0,1, \ldots, n-1$ we have a short exact sequence of $\mathrm{C}^{*}$-algebras

$$
0 \longrightarrow C^{*}\left(G, I_{k}, \alpha\right) \longrightarrow C^{*}\left(G, I_{k+1}, \alpha\right) \longrightarrow C^{*}\left(G, I_{k+1} / I_{k}, \alpha\right) \longrightarrow 0 .
$$

Lemma 4.6 implies that $C^{*}\left(G, I_{k+1} / I_{k}, \alpha\right)$ has finite primitive ideal space. Moreover, if $A$ is purely infinite, then $I_{k+1} / I_{k}$ is purely infinite by Theorem 4.19 of [13], so Lemma 4.6 implies that $C^{*}\left(G, I_{k+1} / I_{k}, \alpha\right)$ is also purely infinite.

An induction argument using Lemma 4.2 now shows that $\operatorname{Prim}\left(C^{*}(G, A, \alpha)\right)$ is finite. If $A$ is purely infinite, using Theorem 4.19 of [13] in the induction argument shows that then $C^{*}(G, A, \alpha)$ is also purely infinite. 
Corollary 4.8. Let $\alpha: G \rightarrow \operatorname{Aut}(A)$ be an action of a finite group $G$ on a $C^{*}$ algebra $A$. If $A$ is purely infinite and has finitely many $\alpha$-invariant ideals, then $C^{*}(G, A, \alpha)$ has the ideal property.

Proof. By Theorem 4.7, the algebra $C^{*}(G, A, \alpha)$ is purely infinite and has finite primitive ideal space. Proposition 2.3 of [15] therefore implies that $C^{*}(G, A, \alpha)$ has the ideal property.

Corollary 4.9. Let $\alpha: G \rightarrow \operatorname{Aut}(A)$ be an action of a finite group $G$ on a $C^{*}$ algebra $A$. Assume that $A$ has finitely many $\alpha$-invariant ideals. Then $\operatorname{Prim}\left(A^{\alpha}\right)$ is finite. Moreover, if in addition $A$ is purely infinite, then $A^{\alpha}$ is purely infinite.

Proof. Theorem 4.7 gives the conclusions for $C^{*}(G, A, \alpha)$. Now apply Proposition 0.1 . The first conclusion follows from the fact that the primitive ideal space of a corner is a subset of the primitive ideal space of the original algebra. The second conclusion follows from the fact (Proposition 4.17 of [13]) that hereditary subalgebras of purely infinite algebras are again purely infinite.

Remark 4.10. There are a fair number of $\mathrm{C}^{*}$-algebras which are purely infinite and have finite primitive ideal spaces, as required in Theorem 4.7 and Corollary 4.9.

For example, this is true of the $\mathrm{C}^{*}$-algebras of many finite graphs. If a finite graph $E$ satisfies Condition $(\mathrm{K})$ as defined before Examples 4.6 of [27], then $\operatorname{Prim}\left(C^{*}(E)\right)$ is finite. Necessary and sufficient conditions for pure infiniteness of $C^{*}(E)$ are given in Theorem 2.3 of [10]. (We are grateful to Gene Abrams for help in locating this reference.) In particular, the conditions in parts (d) and (e) of that theorem are entirely in terms of the graph. (Warning: the convention relating the direction of the arrows in a graph $E$ to the definition of $C^{*}(E)$ in [10] is the opposite of the convention in [27.)

Theorem 4.7 and Corollary 4.9 also apply to many minimal tensor products $B \otimes_{\min } D$ in which $B$ is purely infinite simple and $\operatorname{Prim}(D)$ is finite. For example, it is enough to assume that $B$ is exact (which ensures that $\operatorname{Prim}\left(B \otimes_{\min } D\right) \cong \operatorname{Prim}(D)$ when $\operatorname{Prim}(D)$ is finite) and approximately divisible (so that Theorem 4.5 of [13] applies).

We can get a little farther by considering composition series.

Theorem 4.11. Let $A$ be a purely infinite $C^{*}$-algebra. Suppose there is an ordinal $\kappa$ and a composition series $\left(I_{\lambda}\right)_{\lambda \leq \kappa}$ for $A$ such that $\operatorname{Prim}\left(I_{\lambda+1} / I_{\lambda}\right)$ is finite for all $\lambda<\kappa$. Let $G$ be a finite group, and let $\alpha: G \rightarrow \operatorname{Aut}(A)$ be any action of $G$ on $A$. Then $C^{*}(G, A, \alpha)$ and $A^{\alpha}$ are purely infinite and have composition series in which all the subquotients have finite primitive ideal spaces.

Proof. We first claim that $A$ has a $G$-invariant composition series $\left(J_{\lambda}\right)_{\lambda \leq \kappa}$ such that $\operatorname{Prim}\left(J_{\lambda+1} / J_{\lambda}\right)$ is finite for all $\lambda<\kappa$. For use in this argument, for an ideal $I$ in a $\mathrm{C}^{*}$-algebra $B$ with an action $\beta: G \rightarrow \operatorname{Aut}(B)$, we write $G I$ for the ideal $\sum_{g \in G} \beta_{g}(I)$. Define $J_{\lambda}=G I_{\lambda}$ for $\lambda \leq \kappa$. It is easy to check that $\left(J_{\lambda}\right)_{\lambda \leq \kappa}$ is a $G$ invariant composition series. (Some of the subquotients may be zero, but this does not matter.) So we must show that, for $\lambda<\kappa$, the space $\operatorname{Prim}\left(\left(G I_{\lambda+1}\right) /\left(G I_{\lambda}\right)\right)$ is finite. First, we have

$$
\left(G I_{\lambda}+I_{\lambda+1}\right) /\left(G I_{\lambda}\right) \cong I_{\lambda+1} /\left(I_{\lambda+1} \cap G I_{\lambda}\right) .
$$


Applying Lemma 4.2 with $I_{\lambda+1} / I_{\lambda}$ in place of $A$, we see that this algebra has finite primitive ideal space. As ideals in $A /\left(G I_{\lambda}\right)$, we have

$$
\left(G I_{\lambda+1}\right) /\left(G I_{\lambda}\right)=G\left(\left(G I_{\lambda}+I_{\lambda+1}\right) /\left(G I_{\lambda}\right)\right)=\sum_{g \in G} \alpha_{g}\left(\left(G I_{\lambda}+I_{\lambda+1}\right) /\left(G I_{\lambda}\right)\right),
$$

which has finite primitive ideal space by Corollary 4.3. This completes the proof of the claim.

It now follows from Theorem 4.7 that $C^{*}(G, A, \alpha)$ has a composition series in which all the subquotients have finite primitive ideal spaces. It easily follows from Proposition 0.1 that the same is true for $A^{\alpha}$.

We now prove by induction on $\lambda$ that $C^{*}\left(G, J_{\lambda}, \alpha\right)$ is purely infinite, starting with the smallest $\lambda_{0}$ such that $J_{\lambda_{0}} \neq\{0\}$. Theorem 4.19 of 13 implies that $J_{\lambda_{0}}$ is purely infinite, and by construction $\operatorname{Prim}\left(J_{\lambda_{0}}\right)$ is finite, so $C^{*}\left(G, J_{\lambda_{0}}, \alpha\right)$ is purely infinite by Theorem 4.7.

Suppose now that $C^{*}\left(G, J_{\mu}, \alpha\right)$ is known to be purely infinite for all $\mu<\lambda$. If there is $\mu$ such that $\mu+1=\lambda$, then we have a short exact sequence

$$
0 \longrightarrow C^{*}\left(G, J_{\mu}, \alpha\right) \longrightarrow C^{*}\left(G, J_{\mu+1}, \alpha\right) \longrightarrow C^{*}\left(G, J_{\mu+1} / J_{\mu}, \alpha\right) \longrightarrow 0,
$$

in which $C^{*}\left(G, J_{\mu}, \alpha\right)$ is purely infinite by the induction hypothesis and $C^{*}\left(G, J_{\mu+1} / J_{\mu}, \alpha\right)$ is purely infinite by the same argument as for $\lambda=\lambda_{0}$. Therefore $C^{*}\left(G, J_{\mu+1}, \alpha\right)$ is purely infinite by Theorem 4.19 of [13].

If $\lambda$ is a limit ordinal, then

$$
C^{*}\left(G, J_{\lambda}, \alpha\right)=\overline{\bigcup_{\mu<\lambda} C^{*}\left(G, J_{\mu}, \alpha\right)}
$$

which is a direct limit of purely infinite $\mathrm{C}^{*}$-algebras by the induction hypothesis, and therefore is purely infinite by Proposition 4.18 of 13 .

This completes the induction. Thus $C^{*}(G, A, \alpha)$ is purely infinite. For $A^{\alpha}$, apply Proposition 4.17 of 13 and Proposition 0.1

We give an example for Theorem 4.11 in which the $\mathrm{C}^{*}$-algebra is not a direct sum of $\mathrm{C}^{*}$-algebras with finite primitive ideal spaces.

Example 4.12. Adopt the conventions for graph $\mathrm{C}^{*}$-algebras described in Chapter 1 of [27]. (Warning: the papers [1], 10], and [14, all used below, use the opposite convention for the relation between the direction of the arrows in the graph and the definition of its $\mathrm{C}^{*}$-algebra.) Consider the following graph $E$ :

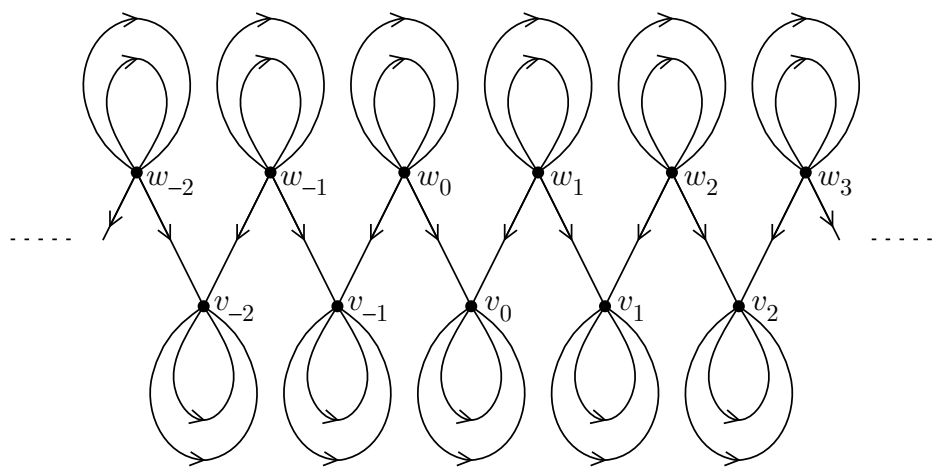


Let $C^{*}(E)$ be its $C^{*}$-algebra. The automorphism group of $E$ acts on $C^{*}(E)$, as described, for example, in the introduction to Section 3 of [14. There is a unique automorphism $h$ of $E$ of order 2 such that $h\left(v_{n}\right)=v_{-n}$ and $h\left(w_{n}\right)=w_{1-n}$ for all $n \in \mathbb{Z}$, and which sends the outer loop at each vertex $x$ to the outer loop at $h(x)$. This gives an automorphism $\alpha$ of $C^{*}(E)$ of order 2 .

We now check the properties of $C^{*}(E)$. We can check pure infiniteness using Theorem 2.3 of [10], but for this graph it seems easier to proceed more directly. The graph $E$ satisfies Condition (K) as defined before Examples 4.6 of [27] and is row finite. Therefore Theorem 4.9 of [27] applies. The set $H=\left\{w_{n}: n \in \mathbb{Z}\right\}$ is a saturated hereditary subset of the vertices, so this theorem gives, using the notation there, a short exact sequence

$$
0 \longrightarrow I_{H} \longrightarrow C^{*}(E) \longrightarrow C^{*}(E \backslash H) \longrightarrow 0,
$$

in which $I_{H}$ has a full corner isomorphic to $C^{*}\left(E_{H}\right)$. Both $E \backslash H$ and $E_{H}$ are isomorphic to countable disjoint unions of copies of the graph with one vertex and two edges, whose $\mathrm{C}^{*}$-algebra is $\mathcal{O}_{2}$. Therefore $C^{*}(E \backslash H) \cong \bigoplus_{n \in \mathbb{Z}} \mathcal{O}_{2}$ and $I_{H}$ is stably isomorphic to $\bigoplus_{n \in \mathbb{Z}} \mathcal{O}_{2}$. It now follows from Theorem 4.19 of [13] that $C^{*}(E)$ is purely infinite.

The graph $E$ is not a disjoint union of nonempty graphs, from which it is easy to see that $C^{*}(E)$ does not have a nontrivial direct sum decomposition. The sets

$$
H_{n}=\left\{v_{-n}, v_{-n+1}, \ldots, v_{n-1}, w_{-n}, w_{-n+1}, \ldots, w_{n}\right\},
$$

for $n \in \mathbb{Z}_{\geq 0}$, form an increasing sequence of finite saturated hereditary subsets of the vertices of $E$ whose union is all the vertices of $E$. The corresponding ideals each have finitely many ideals (using Theorem 4.19 of [13]). Their union is dense in $C^{*}(E)$, since, by Theorem 4.19 of [13], the closure of the union comes from some saturated hereditary subset of the vertices of $E$. Therefore $C^{*}(E)$ has a composition series in which all the subquotients have finite primitive ideal spaces.

We didn't explicitly need the space $\operatorname{Prim}\left(C^{*}(E)\right)$, but it can easily be calculated using Theorem 6.3 of [1].

There are many other automorphisms of the graph $E$ in Example 4.12 of order two. For example, one can modify $h$ by having it exchange the two loops at $v_{0}$ instead of acting trivially on them. One similarly gets two automorphisms of order two which act on the vertices via $v_{n} \mapsto v_{-n-1}$ and $w_{n} \mapsto w_{-n}$. One gets automorphisms of order four by specifying that for some values of $n>0$, the automorphism should send the outer loop at $v_{n}$ or at $w_{n}$ to the inner loop at $h\left(v_{n}\right)$ or at $h\left(w_{n}\right)$.

Free actions on graphs are the subject of a very nice theorem in [14, and we can make a similar example with a free action of $\mathbb{Z}_{2}$. Let $F$ be the following graph:

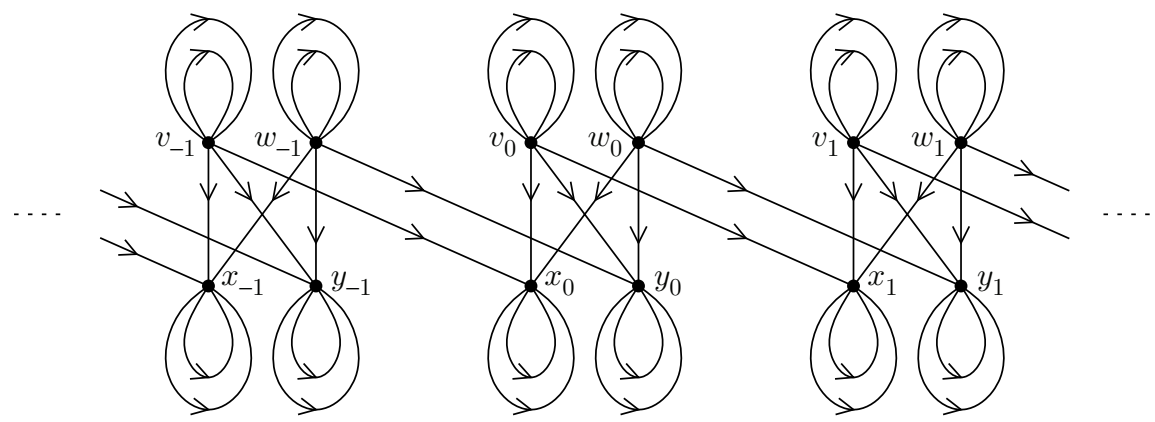


Then $C^{*}(F)$ is purely infinite and has a composition series whose subquotients have finite primitive ideal spaces by the same reasoning as in Example 4.12. There is an automorphism $h: F \rightarrow F$ of order 2 which acts on the vertices by

$$
h\left(v_{n}\right)=w_{n}, \quad h\left(w_{n}\right)=v_{n}, \quad h\left(x_{n}\right)=y_{n}, \quad \text { and } \quad h\left(y_{n}\right)=x_{n}
$$

for $n \in \mathbb{Z}$, and the corresponding action of $\mathbb{Z}_{2}$ on $F$ is free.

A proof related to that of Theorem 4.11 also gives the following result. The hypotheses are stronger than those of Theorem 4.11, but still apply to a $\mathrm{C}^{*}$-algebra which is an arbitrary (not necessarily finite) direct sum of purely infinite $\mathrm{C}^{*}$-algebras with finite primitive ideal spaces, and to a purely infinite $\mathrm{C}^{*}$-algebra with a composition series indexed by $\mathbb{Z}_{>0}$ and with simple subquotients. Example 4.12 and the related examples discussed afterwards also satisfy its hypotheses. The conclusion is stronger, since it includes the ideal property for both the crossed product and the fixed point algebra. In particular, pure infiniteness rules out the phenomenon in Example 2.7.

Proposition 4.13. Let $\alpha: G \rightarrow \operatorname{Aut}(A)$ be an action of a finite group $G$ on a $C^{*}$-algebra A. Suppose that there is a set $\mathcal{I}$ of ideals in $A$, each of which is purely infinite and has finite primitive ideal space, with the following property. For every finite subset $S \subset A$ and every $\varepsilon>0$, there is $I \in \mathcal{I}$ such that $\operatorname{dist}(a, I)<\varepsilon$ for all $a \in S$. Then $C^{*}(G, A, \alpha)$ and $A^{\alpha}$ are purely infinite and have the ideal property.

Proof. We use the notation GI from the proof of Theorem 4.11. Let $\Lambda$ be the set of all finite subsets of $\mathcal{I}$. For $\lambda \in \Lambda$, set $J_{\lambda}=\sum_{I \in \lambda} G I$. Then $\operatorname{Prim}\left(J_{\lambda}\right)$ is finite by Corollary 4.3. An argument similar to one used in the proof of Theorem 4.11 shows that $C^{*}\left(G, J_{\lambda}, \alpha\right)$ is purely infinite, so Proposition 4.17 of [13] and Proposition 0.1 imply that $\left(J_{\lambda}\right)^{\alpha}$ is purely infinite. Theorem 4.7 also implies that $\operatorname{Prim}\left(C^{*}\left(G, J_{\lambda}, \alpha\right)\right)$ is finite, so Proposition 0.1 implies that $\operatorname{Prim}\left(\left(J_{\lambda}\right)^{\alpha}\right)$ is finite. So $C^{*}\left(G, J_{\lambda}, \alpha\right)$ and $\left(J_{\lambda}\right)^{\alpha}$ have the ideal property by Proposition 2.3 of 15 .

We have $A=\lim _{\lambda \in \Lambda} J_{\lambda}$, so $C^{*}(G, A, \alpha)=\lim _{\lambda \in \Lambda} C^{*}\left(G, J_{\lambda}, \alpha\right)$, which is then purely infinite by Proposition 4.18 of [13]. Also, $C^{*}(G, A, \alpha)$ has the ideal property by Proposition 2.3 of [18. Since $A^{\alpha}=\varliminf_{\lambda \in \Lambda}\left(J_{\lambda}\right)^{\alpha}$, the same reasoning shows that $A^{\alpha}$ is purely infinite and has the ideal property.

\section{REFERENCES}

[1] Teresa Bates, David Pask, Iain Raeburn, and Wojciech Szymański, The $C^{*}$-algebras of rowfinite graphs, New York J. Math. 6 (2000), 307-324 (electronic). MR 1777234 (2001k:46084)

[2] Bruce Blackadar, K-theory for operator algebras, 2nd ed., Mathematical Sciences Research Institute Publications, vol. 5, Cambridge University Press, Cambridge, 1998. MR.1656031 (99g:46104)

[3] Ola Bratteli and George A. Elliott, Structure spaces of approximately finite-dimensional $C^{*}$ algebras. II, J. Funct. Anal. 30 (1978), no. 1, 74-82, DOI 10.1016/0022-1236(78)90056-3. MR.513479 (80d:46111)

[4] Lawrence G. Brown and Gert K. Pedersen, Limits and $C^{*}$-algebras of low rank or dimension, J. Operator Theory 61 (2009), no. 2, 381-417. MR2501012 (2011f:46065)

[5] José R. Carrión and Cornel Pasnicu, Approximations of $C^{*}$-algebras and the ideal property, J. Math. Anal. Appl. 338 (2008), no. 2, 925-945, DOI 10.1016/j.jmaa.2007.05.074. MR2386471 (2009j:46123)

[6] Marius Dadarlat and Cornel Pasnicu, Continuous fields of Kirchberg $C^{*}$-algebras, J. Funct. Anal. 226 (2005), no. 2, 429-451, DOI 10.1016/j.jfa.2005.01.005. MR2160103 (2006g:46098)

[7] Jacques Dixmier, $C^{*}$-algebras, North-Holland Publishing Co., Amsterdam, 1977. Translated from the French by Francis Jellett; North-Holland Mathematical Library, Vol. 15. MR.0458185 (56 \#16388) 
[8] Elliot C. Gootman, Aldo J. Lazar, and Costel Peligrad, Spectra for compact group actions, J. Operator Theory 31 (1994), no. 2, 381-399. MR.1331784 (96f:46117)

[9] Philip Green, The structure of imprimitivity algebras, J. Funct. Anal. 36 (1980), no. 1, 88104, DOI 10.1016/0022-1236(80)90108-1. MR568977 (83d:46080)

[10] Jeong Hee Hong and Wojciech Szymański, Purely infinite Cuntz-Krieger algebras of directed graphs, Bull. London Math. Soc. 35 (2003), no. 5, 689-696, DOI 10.1112/S0024609303002364. MR.1989499 (2005c:46097)

[11] Masaki Izumi, Finite group actions on $C^{*}$-algebras with the Rohlin property. I, Duke Math. J. 122 (2004), no. 2, 233-280, DOI 10.1215/S0012-7094-04-12221-3. MR2053753 (2005a:46142)

[12] Ja A Jeong and Hiroyuki Osaka, Extremally rich $C^{*}$-crossed products and the cancellation property, J. Austral. Math. Soc. Ser. A 64 (1998), no. 3, 285-301. MR1623278 (99k:46097)

[13] Eberhard Kirchberg and Mikael Rørdam, Non-simple purely infinite $C^{*}$-algebras, Amer. J. Math. 122 (2000), no. 3, 637-666. MR.1759891 (2001k:46088)

[14] Alex Kumjian and David Pask, $C^{*}$-algebras of directed graphs and group actions, Ergodic Theory Dynam. Systems 19 (1999), no. 6, 1503-1519, DOI 10.1017/S0143385799151940. MR:1738948(2000m:46125)

[15] Dan Kucerovsky, P. W. Ng, and Francesc Perera, Purely infinite corona algebras of simple $C^{*}$ algebras, Math. Ann. 346 (2010), no. 1, 23-40, DOI 10.1007/s00208-009-0382-0. MR2558884 (2010j:46117)

[16] Gerard J. Murphy, $C^{*}$-algebras and operator theory, Academic Press Inc., Boston, MA, 1990. MR.1074574 (91m:46084)

[17] Hiroyuki Osaka and N. Christopher Phillips, Crossed products by finite group actions with the Rokhlin property, Math. Z. 270 (2012), no. 1-2, 19-42, DOI 10.1007/s00209-010-0784-4. MR 2875821

[18] Cornel Pasnicu, On the AH algebras with the ideal property, J. Operator Theory 43 (2000), no. 2, 389-407. MR1753416 (2001c:46111)

[19] Cornel Pasnicu, Ideals generated by projections and inductive limit $C^{*}$-algebras, Rocky Mountain J. Math. 31 (2001), no. 3, 1083-1095, DOI 10.1216/rmjm/1020171681. MR.1877336 (2003f:46087)

[20] Cornel Pasnicu, The projection property, Glasg. Math. J. 44 (2002), no. 2, 293-300, DOI 10.1017/S0017089502020104. MR1902406 (2003i:46058)

[21] Cornel Pasnicu, LB algebras, J. Operator Theory 50 (2003), no. 2, 263-281. MR2050129 (2005e:46101)

[22] Cornel Pasnicu and Mikael Rørdam, Purely infinite $C^{*}$-algebras of real rank zero, J. Reine Angew. Math. 613 (2007), 51-73, DOI 10.1515/CRELLE.2007.091. MR2377129 (2009b:46119)

[23] Gert K. Pedersen, $C^{*}$-algebras and their automorphism groups, London Mathematical Society Monographs, vol. 14, Academic Press Inc. [Harcourt Brace Jovanovich Publishers], London, 1979. MR548006 (81e:46037)

[24] N. Christopher Phillips, Equivariant K-theory and freeness of group actions on $C^{*}$ algebras, Lecture Notes in Mathematics, vol. 1274, Springer-Verlag, Berlin, 1987. MR.911880 (89k:46086)

[25] N. Christopher Phillips, The tracial Rokhlin property for actions of finite groups on $C^{*}$-algebras, Amer. J. Math. 133 (2011), no. 3, 581-636, DOI 10.1353/ajm.2011.0016. MR:2808327(2012h:46116)

[26] N. Christopher Phillips, Freeness of actions of finite groups on $C^{*}$-algebras, Operator structures and dynamical systems, Contemp. Math., vol. 503, Amer. Math. Soc., Providence, RI, 2009, pp. 217-257, DOI 10.1090/conm/503/09902. MR2590625(2012f:46143)

[27] Iain Raeburn, Graph algebras, CBMS Regional Conference Series in Mathematics, vol. 103. Published for the Conference Board of the Mathematical Sciences, Washington, DC, 2005. MR2135030 (2005k:46141)

[28] Marc A. Rieffel, Actions of finite groups on $C^{*}$-algebras, Math. Scand. 47 (1980), no. 1, 157-176. MR600086 (83c:46062)

[29] J. Rosenberg, Appendix to O. Bratteli's paper on "Crossed products of UHF algebras", Duke Math. J. 46(1979), 25-26.

[30] Mikael Rørdam and Adam Sierakowski, Purely infinite $C^{*}$-algebras arising from crossed products, Ergodic Theory Dynam. Systems 32 (2012), no. 1, 273-293, DOI 10.1017/S0143385710000829. MR2873171 (2012m:46063) 
[31] Adam Sierakowski, The ideal structure of reduced crossed products, Münster J. Math. 3 (2010), 237-261. MR2775364 (2012g:46103)

[32] Kenneth H. Stevens, The classification of certain non-simple approximate interval algebras, Operator algebras and their applications, II (Waterloo, ON, 1994/1995), Fields Inst. Commun., vol. 20, Amer. Math. Soc., Providence, RI, 1998, pp. 105-148. MR.1643184 (2002d:46052)

[33] Shuang Zhang, On the structure of projections and ideals of corona algebras, Canad. J. Math. 41 (1989), no. 4, 721-742, DOI 10.4153/CJM-1989-033-4. MR.1012625 (90h:46094)

[34] Shuang Zhang, A Riesz decomposition property and ideal structure of multiplier algebras, J. Operator Theory 24 (1990), no. 2, 209-225. MR.1150618 (93b:46116)

Department of Mathematics, The University of Texas at San Antonio, San Antonio, TEXAS 78249

E-mail address: Cornel.Pasnicu@utsa.edu

Department of Mathematics, University of Oregon, Eugene, Oregon 97403-1222

E-mail address: ncp@darkwing.uoregon.edu 